\title{
Design and Experimental Investigation of Rotational Angle Based Tracking Control
}

\author{
A THESIS \\ SUBMITTED TO THE FACULTY OF THE GRADUATE SCHOOL \\ OF THE UNIVERSITY OF MINNESOTA \\ BY
}

Meng Yang

IN PARTIAL FULFILLMENT OF THE REQUIREMENTS

FOR THE DEGREE OF

MASTER OF SCIENCE

Dr. Zongxuan Sun, Advisor

August, 2014 
(C) Meng Yang 2014

ALL RIGHTS RESERVED 


\section{Acknowledgements}

It is with great honor that I would like to thank my advisor, Professor Zongxuan Sun, for allowing me to work on the exciting research project for my Master's thesis. His deeper insights and his innovative ideas opened my eyes to the many challenges that both the life and the industry face. Without his continuous support, I can sincerely say that I would not be here writing this today.

I would also like to thank Dr. Rajesh Rajamani and Dr. Peter Seiler Jr for accepting my request to serve as my examining committee. I would like to extend my many thanks to the whole research team --Dr. Xingyong Song, Dr. Pradeep Gillella, Dr. Yu Wang, Yongsoon Yoon, ChienShin Wu, Ke Li, Chen Zhang, Azrin Mohd Zulkefli, Yaoying Wang and Virinchi Mallela in all kind of assistants in my graduate study. I would also like to acknowledge Dr. Xingyong Song for his diligence in all the works that we had done together.

Last but not least, I would like to express my greatest gratitude to my parents, Yuheng Zhang and Jiannong Yang, for their long-lasting support throughout my life and study. Their unconditional love provides me constant encouragement and strength whenever challenges arose. 


\section{Dedication}

To my family and friends. 


\begin{abstract}
This work investigates the tracking control in the rotational angle domain based on the time-varying internal model principle. The focus is to enable precise, reliable and computational efficient output tracking/disturbance rejection in the angle domain. To achieve better performance, existing approaches typically require more discrete samplings per revolution, which can drastically increase the controller order and also poses challenge for the stabilizer convergence. To address those issues, a varying sampling interval approach is proposed, where the control sampling rate is not fixed but optimized based on errors between sampling points, so that proper regulation performance can be achieved without significantly increasing the number of sampling points. Meanwhile, to improve the convergence rate of the tracking error, additional LMI constraints are added to the existing stabilizer synthesis. Through experimental study on a camless engine valve actuation system, the effectiveness of the proposed approaches is well demonstrated.
\end{abstract}




\section{Contents}

Acknowledgements i

Dedication ii

Abstract iii

List of Tables vi vi vis

List of Figures vii

$\begin{array}{lll}\text { Chapter } 1 & \text { Introduction and Background } & 1\end{array}$

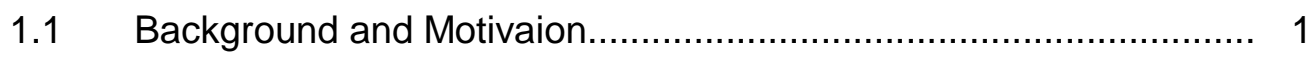

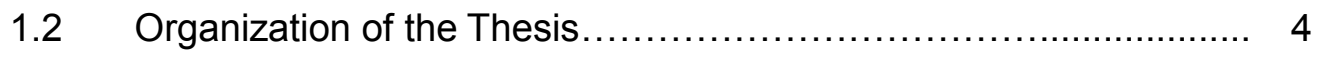

Chapter 2 Convergence Improvement for Angle Varying Gain Based Stabilizer Design $\quad 6$

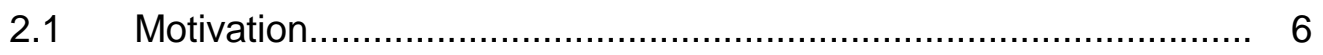

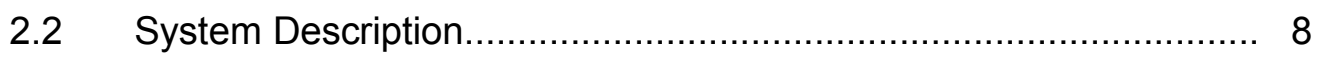

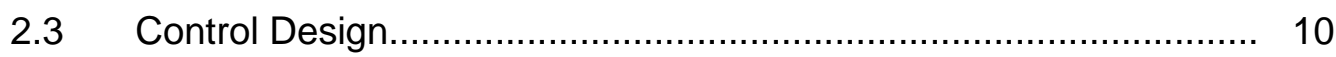

2.4 Convergence Regulation for Angle Varying Gain Based Stabilizer Design................................................................................ 12

$2.5 \quad$ Simulation Results.................................................... 15

2.6 Conclusion....................................................................... 18

Chapter $3 \quad$ Variable Sampling in the Angle Domain 19

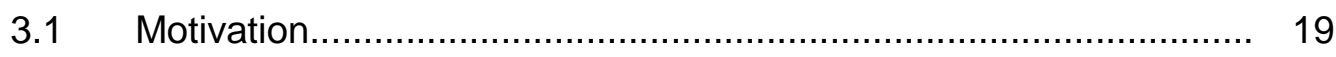

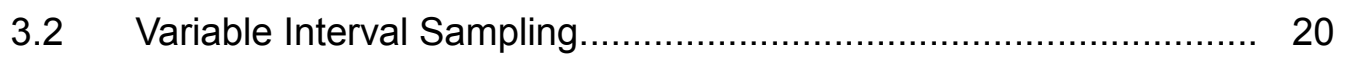


3.3 An Iterative Approach for Searching Optimal Sampling Interval.... 21

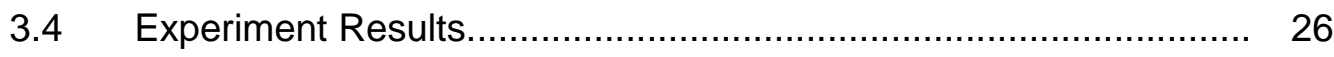

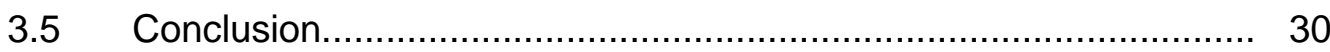

\section{$\begin{array}{lll}\text { Chapter } 4 & \text { Experimental Investigations } & 31\end{array}$}

4.1 Camless Engine Valve Hardware Setup................................. 31

4.2 Experiment Validation for Convergence-improved Stabilizer.......... 34

4.2.1 12-point fixed interval sampling case....................... 34

4.2.2 14-point fixed interval sampling case...................... 36

4.2.3 16-point fixed interval sampling case.................... 38

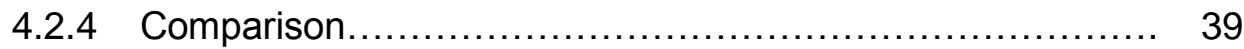

4.3 Experiment Validation for Variable Sampling Interval Searching..... 40

4.3.1 14-point variable interval sampling case ................... 40

4.3.2 10-point variable interval sampling case.................... 41

4.3.3 8-point variable interval sampling case $\ldots \ldots \ldots \ldots \ldots \ldots \ldots \ldots . \ldots 3$

4.3.4 6-point and 4-point variable interval sampling case.......... 45

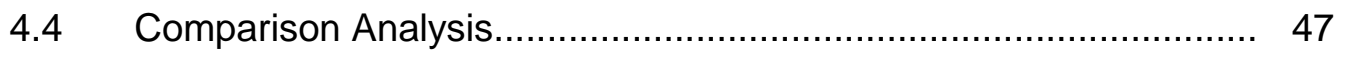

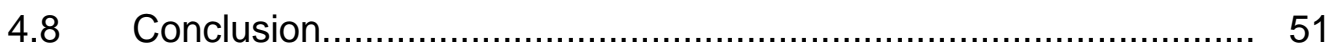

Chapter 5 Conclusion $\quad 52$

References 


\section{List of Tables}

4.1 Comparison of the convergence achieved using the original stabilizer and convergence-improved stabilizer........................... 


\section{List of Figures}

1.1. Signal profile in the angle domain (top) and the time domain (bottom)

2.1. Comparison of convergence rate for system with different internal model orders. 7

2.2. (a) Block diagram of the augmented system.......................... 9

2.2. (b) Block diagram of the internal model based control system......... 10

2.3. Reference profile..................................................... 15

2.4. Simulational tracking results with 16-point sampling $[5-9 \mathrm{~Hz} @$

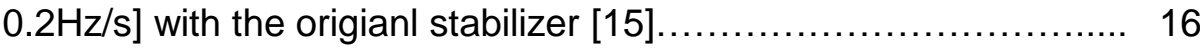

2.5. Simulational tracking results with 16-point sampling $[5-9 \mathrm{~Hz} @$ $0.2 \mathrm{~Hz} / \mathrm{s}]$ with the convergence-improved stabilizer.................... 17

3.1. Comparison of fixed and variable interval sampling......................... 20

3.2. Block diagram of the iterative approach to search the optimal sampling interval for the time-varying internal model based camless engine valve actuation system.

3.3. Initialize sampling intervals of the valve reference

3.4. Flow chart of the iterative approach to search the desired sampling interval.

3.5. Convergence of the varying interval sampling design with $\widetilde{K}_{L}=20 \ldots$

3.6. Convergence of the iterative varying interval sampling searching with $\left[\widetilde{K}_{1}, \widetilde{K}_{2}, \widetilde{K}_{3}\right]=[28,22,20]$

3.7. Experimental tracking results with 12-point variable interval sampling [5-9Hz @ 0.2Hz/s] with the convergence-improved stabilizer

3.8. Experimental tracking results with 12-point fixed interval sampling $[5-9 \mathrm{~Hz} @ 0.2 \mathrm{~Hz} / \mathrm{s}]$ with the original stabilizer 29

4.1. Camless engine valve actuation system setup..... 32 
4.2. Schematic of camless engine valve actuation system.

4.3. Experimental tracking results with 12-point fixed interval sampling $[5-9 \mathrm{~Hz} @ 0.2 \mathrm{~Hz} / \mathrm{s}]$ with the origianl stabilizer.

4.4. Experimental tracking results with 12-point fixed interval sampling $[5-9 \mathrm{~Hz} @ 0.2 \mathrm{~Hz} / \mathrm{s}]$ with the convergence-improved stabilizer. 35

4.5. Experimental tracking results with 14-point fixed interval sampling $[5-9 \mathrm{~Hz} @ 0.2 \mathrm{~Hz} / \mathrm{s}]$ with the origianl stabilizer...

4.6. Experimental tracking results with 14-point fixed interval sampling $[5-9 \mathrm{~Hz} @ 0.2 \mathrm{~Hz} / \mathrm{s}]$ with the convergence-improved stabilizer.

4.7. Experimental tracking results with 16-point fixed interval sampling $[5-9 \mathrm{~Hz} @ 0.2 \mathrm{~Hz} / \mathrm{s}]$ with the convergence-improved stabilizer.

4.8. Experimental tracking results with 14-point variable interval sampling [5-9Hz @ 0.2 Hz/s] with the convergence-improved stabilizer.

4.9. Experimental tracking results with 10-point fixed interval sampling $[5-9 \mathrm{~Hz} @ 0.2 \mathrm{~Hz} / \mathrm{s}]$ with the original stabilizer.

4.10. Experimental tracking results with 10-point variable interval sampling $[5-9 \mathrm{~Hz} @ 0.2 \mathrm{~Hz} / \mathrm{s}]$ with the convergence-improved stabilizer....

4.11. Experimental tracking results with 8-point fixed interval sampling $[5-9 \mathrm{~Hz} @ 0.2 \mathrm{~Hz} / \mathrm{s}]$ with the original stabilizer...

4.12. Experimental tracking results with 8-point variable interval sampling $[5-9 \mathrm{~Hz} @ 0.2 \mathrm{~Hz} / \mathrm{s}]$ with the convergence-improved stabilizer.

4.13. Experimental tracking results with 6-point fixed interval sampling $[5-9 \mathrm{~Hz} @ 0.2 \mathrm{~Hz} / \mathrm{s}]$ with the original stabilizer.

4.14. Experimental tracking results with 6-point variable interval sampling $[5-9 \mathrm{~Hz} @ 0.2 \mathrm{~Hz} / \mathrm{s}]$ with the originl stabilizer

4.15. Experimental tracking results with 4-point fixed interval sampling $[5-9 \mathrm{~Hz} @ 0.2 \mathrm{~Hz} / \mathrm{s}]$ with the originl stabilizer.

4.16. Experimental tracking results with 4-point variable interval sampling $[5-9 \mathrm{~Hz} @ 0.2 \mathrm{~Hz} / \mathrm{s}]$ with the originl stabilizer 
4.17. Comparison of the tracking performance achieved using different

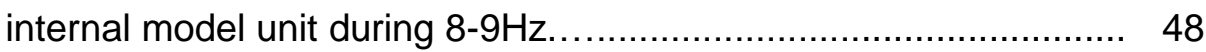

4.18. Experimental tracking results with 8-point fixed interval sampling $[5-20 \mathrm{~Hz} @ 1 \mathrm{~Hz} / \mathrm{s}]$ with the original stabilizer............................... 49

4.19. Experimental tracking results with 8-point variable interval sampling [5-20Hz @ 1Hz/s] with the convergence-improved stabilizer. 50 


\section{Chapter 1}

\section{Introduction and Background}

\subsection{Background and Motivation}

Rotational motion is ubiquitous in engineered systems, ranging from tiny micro actuators [1] to large turbine machines, from medical equipment to household appliances, from mechanical systems (gearbox) to fluid (hydraulic pump/motor) and electrical systems (electrical motor/generator) [2]. These rotational systems inevitably contain angle dependent signal dynamics. Specifically, the signals of interest, such as references to be tracked or disturbances to be rejected, are periodic with respect to the angular displacement.

Precise tracking or rejection of such kind of angle dependent signals could be critical to the rotational systems mentioned above. If the rotational speed is constant, the reference/disturbance is periodic in time domain as well, and the tracking/rejection problem in this case has been well studied. One of the widelyapplied approaches is repetitive control [3-4], where a time-invariant internal model is built by directly embedding the periodic signal generating dynamics in the control loop. Essentially high control gains are invoked at discrete frequency points by the internal model, so that periodic signals, whose frequency spectrum is only non-zero at discrete frequency points, can be regulated in a reliable and accurate fashion. Considering model uncertainties, a Linear Time Invariant (LTI) robust repetitive controller [5] was designed for the constant speed engine operation. However, the LTI repetitive controller is not applicable when the 
reference profile becomes aperiodic in the time domain as the engine speed changes in real-time.
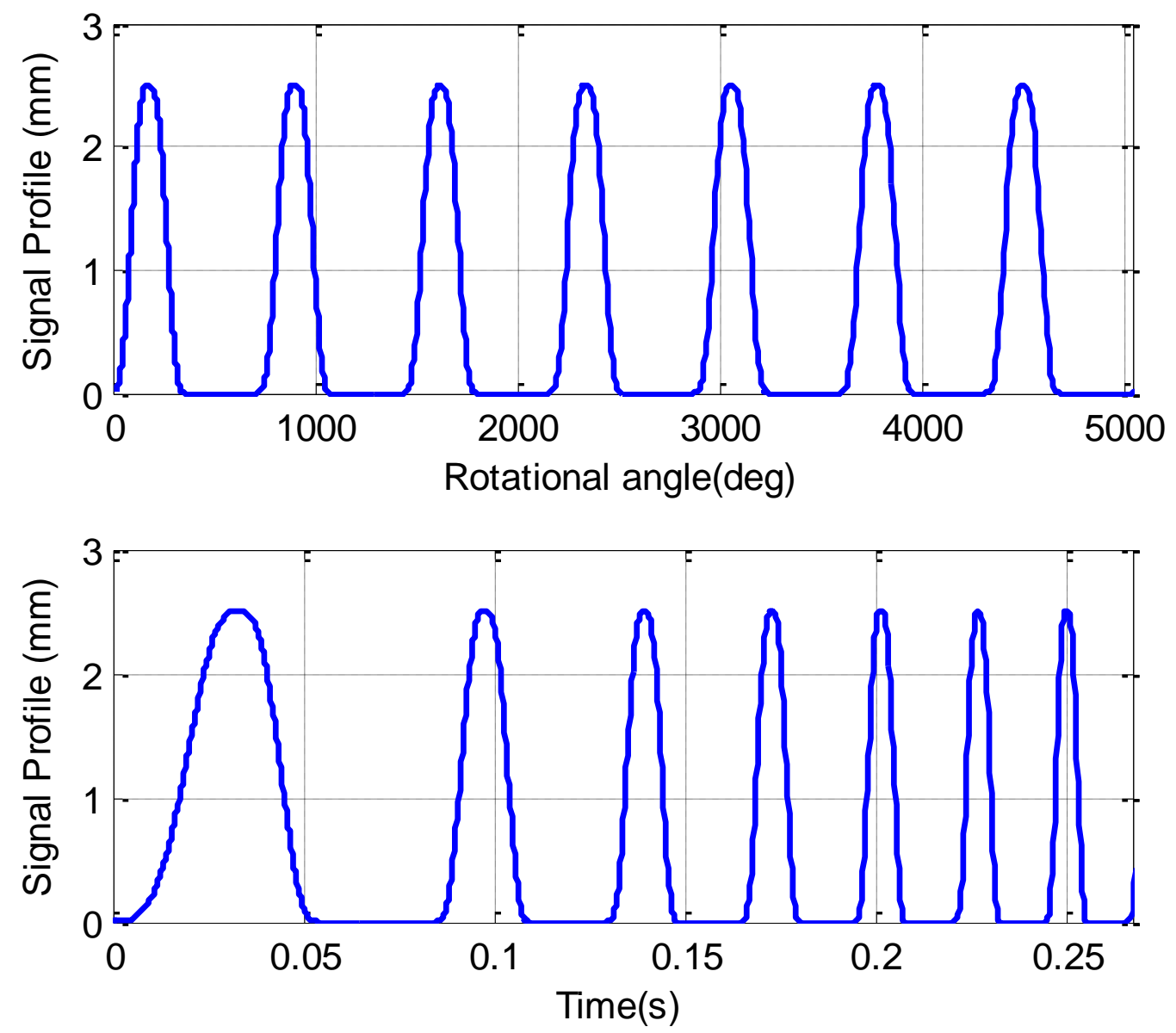

Figure 1.1. Signal profile in the angle domain (top) and the time domain (bottom).

In the case when rotational speed is changing, the reference/disturbance is no longer periodic in the time domain (shown in Figure 1.1). This poses fundamental challenges to the controller design, and repetitive control cannot be applied any more. Motivated by the fact that the signals of interests are still periodic with respect to the angular displacement, control design has been investigated in the angle domain. However, an LTI (Linear Time Invariant) 
system will become angle-varying in the angle domain [9-11] (the rotational speed will show up in the angle domain system dynamics [6-8]), the control design is still non-trivial. One approach is to build the nominal LTI plant by using model reference control design [9] and modify the repetitive controller in real-time. The other approach is to design parameter varying repetitive control [10-11] to track or reject periodic signals in the angle domain while the rotational speed varies in the time domain. Ref [12] introduced an approach by constructing an angle invariant internal model and a linear parameter varying (LPV) stabilizer to stabilize the augmented system (internal model + plant). As pointed out in [13], an angle varying internal model is needed by incorporating both the signal generating dynamics and the angle varying plant dynamics. Ref [14] constructs such an angle dependent controller by leveraging the angle varying internal model structure from [13] and a low order parameter varying stabilizer design proposed in [15-16]. Asymptotic tracking performance is shown in [14] with both simulation and experimental studies, where a number of results from slow to fast rotational speed variation rate are presented to demonstrate the control effectiveness.

One of the challenges of the angle-varying internal model based approach in [14] is the requirement for high sampling rate to achieve better tracking. It is found that fine sampling resolution of the reference profile can improve the tracking performance. One approach is to increase the number of sampling points per revolution in the angle domain. However, this inevitably results in a high order internal model [14], which not only increases the computational cost but also affects the control convergence rate as will be shown in Chapter 2 . These facts limit the potential impact of the angle-based tracking control for practical applications. This work aims at addressing these issues.

First, to improve the control convergence rate, additional LMI constraints are added to the existing stabilizer synthesis in ref [15], which can ensure rapid convergence even with high order internal model. With the benefit of the 
convergence-improved stabilizer, more sampling locations can be applied per revolution to improve the sampling resolution and to obtain better tracking performance.

Secondly, to limit the number of sampling per revolution period while maintaining tracking/rejection preciseness, instead of using a fixed sampling interval, a varying interval sampling approach is proposed to select the sampling location with varying intervals. The idea is to have more sampling points at critical portion of the reference signal, while sampling less at other locations. Moreover, the locations of the sampling points could also be updated based on the tracking results at previous events, where the pattern of the varying sampling interval is adapted while the total number of sampling points for a single rotational cycle stays constant.

\subsection{Organization of the Thesis}

The rest of the document is organized as follows:

Chapter 2 reviews the time-varying internal model based control in the angle domain and presents the convergence-improved stabilizer. The simulation results show a significant improvement in the convergence speed for the high order internal model based system.

Chapter 3 proposes the varying interval sampling method together with rules for sampling interval adaptation. The fixed and varying interval samplings are discussed. Also, an iterative searching method is provided to locate the varying interval sampling points per revolution in the angle domain.

Chapter 4 describes the camless engine valve actuation system. The experimental results are presented to validate the effectiveness and advantage of the proposed methods. The experimental comparison results with different numbers of sampling points per revolution provide a solution to balance the 
complexity of the internal model unit and the precise tracking performance for the application.

Chapter 5 summarizes the research. 


\section{Chapter 2}

\section{Convergence Improvement for Angle Varying Gain Based Stabilizer Design}

\subsection{Motivation}

In the case when rotational speed is changing, the reference is aperiodic in the time domain. The precise motion control is studied by the time-varying internal model based control [14]. However, generating dynamics of the reference in the time domain is dependent on the number of harmonic signals, e.g. $2 N+1$ order dynamic system for the $N$ harmonic signals, and involves more calculations to obtain the coefficients in the generating dynamics. This limits the implementation of the time-varying internal model based control in the time domain for different reference profiles.

Instead of the aperiodic signal with respect to time, the valve reference is periodic in the angle domain. Moreover, Compare with the time-varying generating dynamic in the time domain, the generating dynamics is angleinvariant which can be described as $r=\left(1-z^{-N}\right)^{-1}, N$ is the number of the sampling points. Thus, the generating dynamic in the angle domain is straightforward and independent to the specific signal.

Compared to the time domain, in the angle domain implementation, more advantages are as followed: 1) 1/O representation of the reference profile generating dynamics is straightforward and angle invariant. 2) the internal model 
design is independent to the specific reference profile, which provides the flexibility to track signals with any harmonics; 3 ) the computational burden to design the internal model unit is drastically decreased especially for the high harmonic reference profiles.

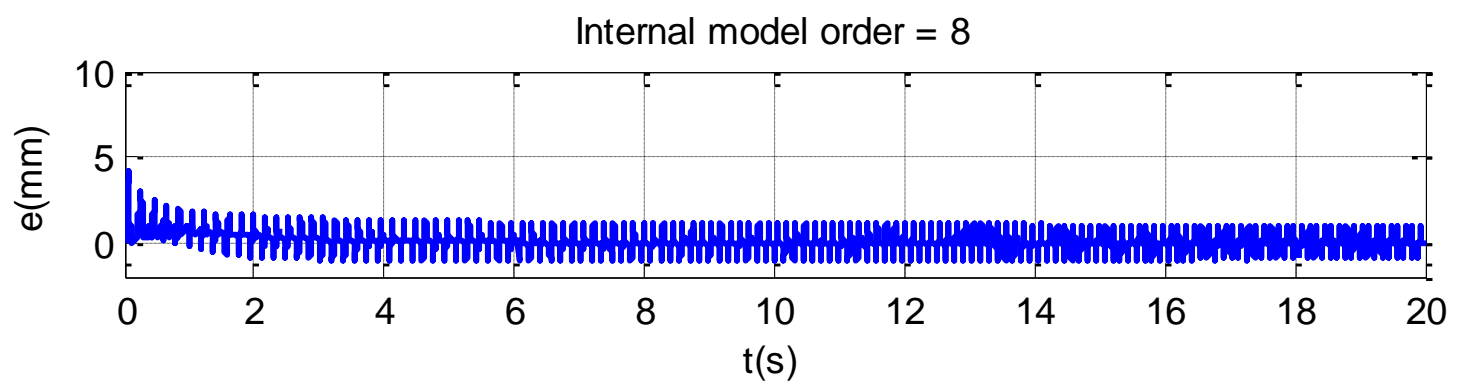

Internal model order $=12$

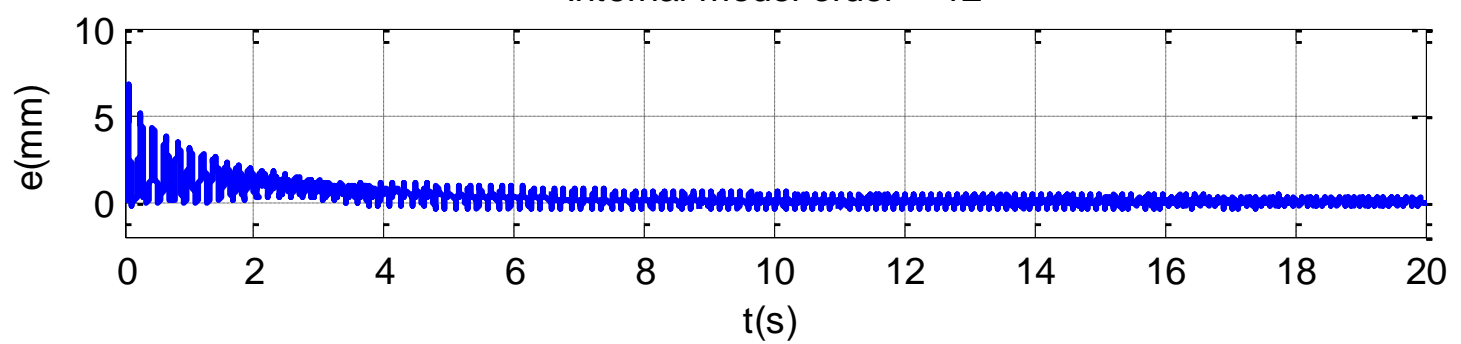

Internal model order $=16$

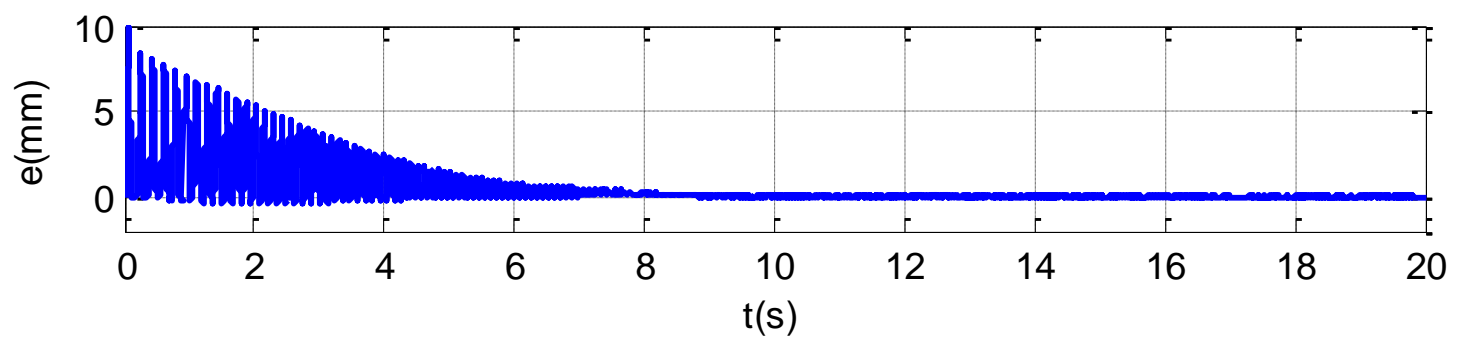

Figure 2.1. Comparison of convergence rate for system with different internal model orders

In order to achieve robust stabilization of the time-varying internal model based control, based on the Lyapunov stability conditions, ref [15] formulates a series of Linear Matrix Inequalities (LMI) for the stabilizer gain synthesis, so that 
the stabilizer gains can be numerically computed through convex optimization. However, it is later found out that directly using LMls given in [15] can result in slow convergence rate when having a high order internal model. This fact can be well observed from simulation results shown in Figure 2.1. It is clearly seen that the convergence becomes slower and the transient magnitude becomes higher with the growth of the internal model order. The main reason is due to the lack of a convergence regulation mechanism for the stabilizer synthesis described in [12].

In order to obtain better tracking performance, fine sampling resolution of the reference profile is required. The most straightforward approach is to locate more sampling points per revolution in the angle domain. However, this inevitably results in a high order internal model [11], which affects the control convergence shown in Figure 2.1. To improve the convergence rate, in this chapter, additional constraints are added to the Lyapunov function, which results in extra terms in the matrix inequalities to complement the synthesis approach described in [15].

\subsection{System Description}

The system plant (Plant $\{1\})$ is a linear time invariant (LTI) system as

$$
\begin{aligned}
& \dot{x}_{c}(t)=A_{c} x_{c}(t)+B_{c} u(t) \\
& y(t)=C_{c} x_{c}(t)
\end{aligned}
$$

where $A_{c} \in R^{n \times n}, B_{c} \in R^{n \times 1}$ and $C_{c} \in R^{1 \times n}$ are the system matrix, input matrix and output matrix, respectively.

Converting the plant dynamics from the time domain into the discrete rotational angle domain, where the discrete sampling is triggered when the angular displacement reaches predetermined values, we have the linear angle varying system in the rotational angle domain [11] as: 


$$
\begin{aligned}
& x_{d}(k+1)=A_{d}(k) x_{d}(k)+B_{d}(k) u(k) \\
& y(k)=C_{d}(k) x_{d}(k)
\end{aligned}
$$

where $A_{d}(k)=e^{A_{c} \Delta T_{k}}, B_{d}(k)=\left[e^{A_{c} \Delta T_{k}}-I\right] A_{c}^{-1} B_{c}, C_{d}(k)=C_{c}, \Delta T_{k}=(\Delta \theta / \omega(k)) \times$ $(60 / 360)$. (Note that the step $k$ refers to the discrete angle step, and $\Delta \theta$ refers to the specific angular displacement.) When the rotational speed $\omega(k)$ changes, the sampling time $\Delta T_{k}$ is not constant and thus $A_{d}(k)$ and $B_{d}(k)$ become anglevarying (dependent on $\omega(k)$ ).

The signal $r$ to be tracked (in the case of reference tracking) or rejected (in the case of disturbance rejection) is periodic with respect to the rotational angle. Its generating dynamics in the angle domain can be described as

$$
\left(1-z^{-N}\right) r=0
$$

where $z^{-1}$ is the one step delay operator, $N$ is the number of the sampling points within a revolution period.

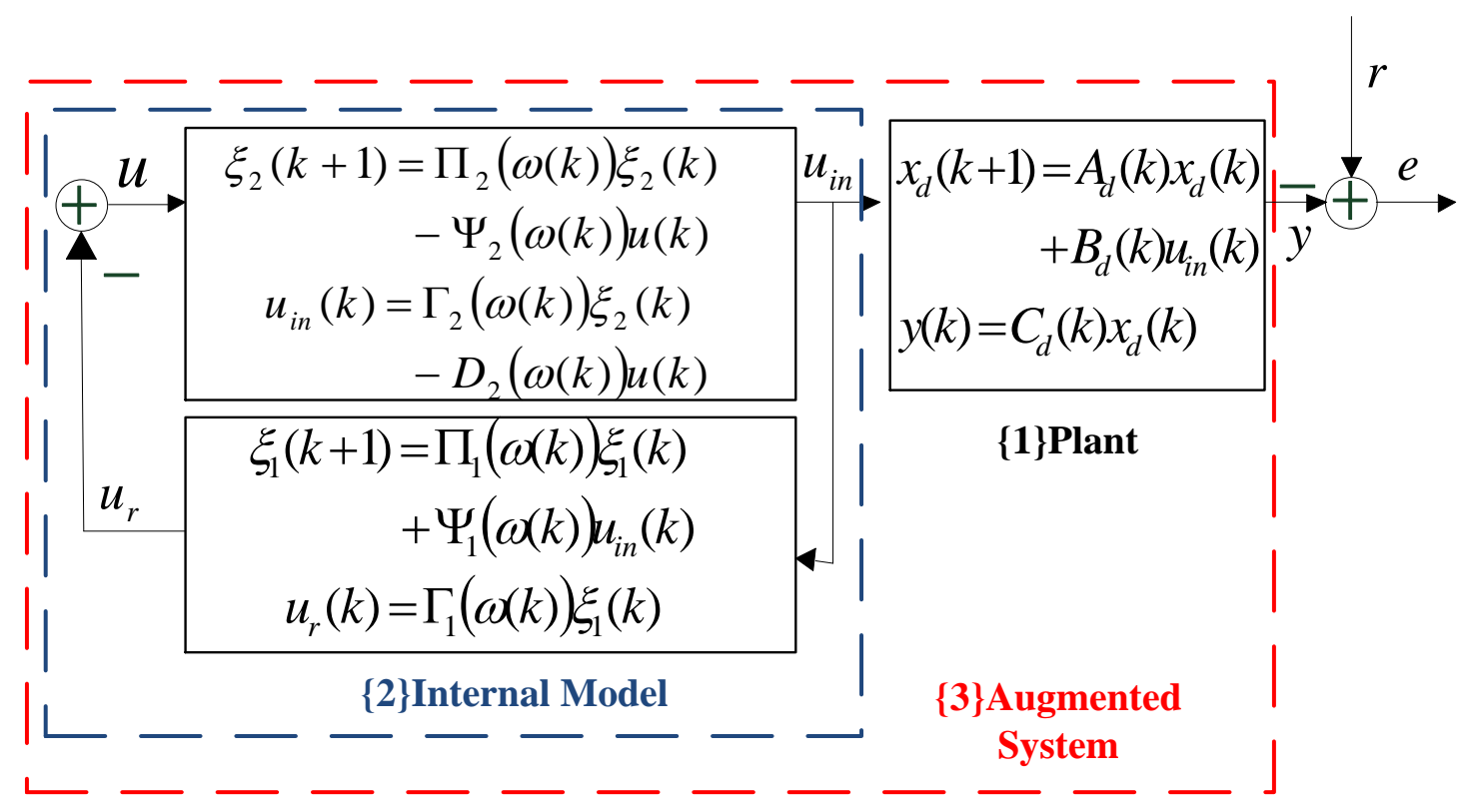

Figure 2.2. (a) Block diagram of the augmented system 


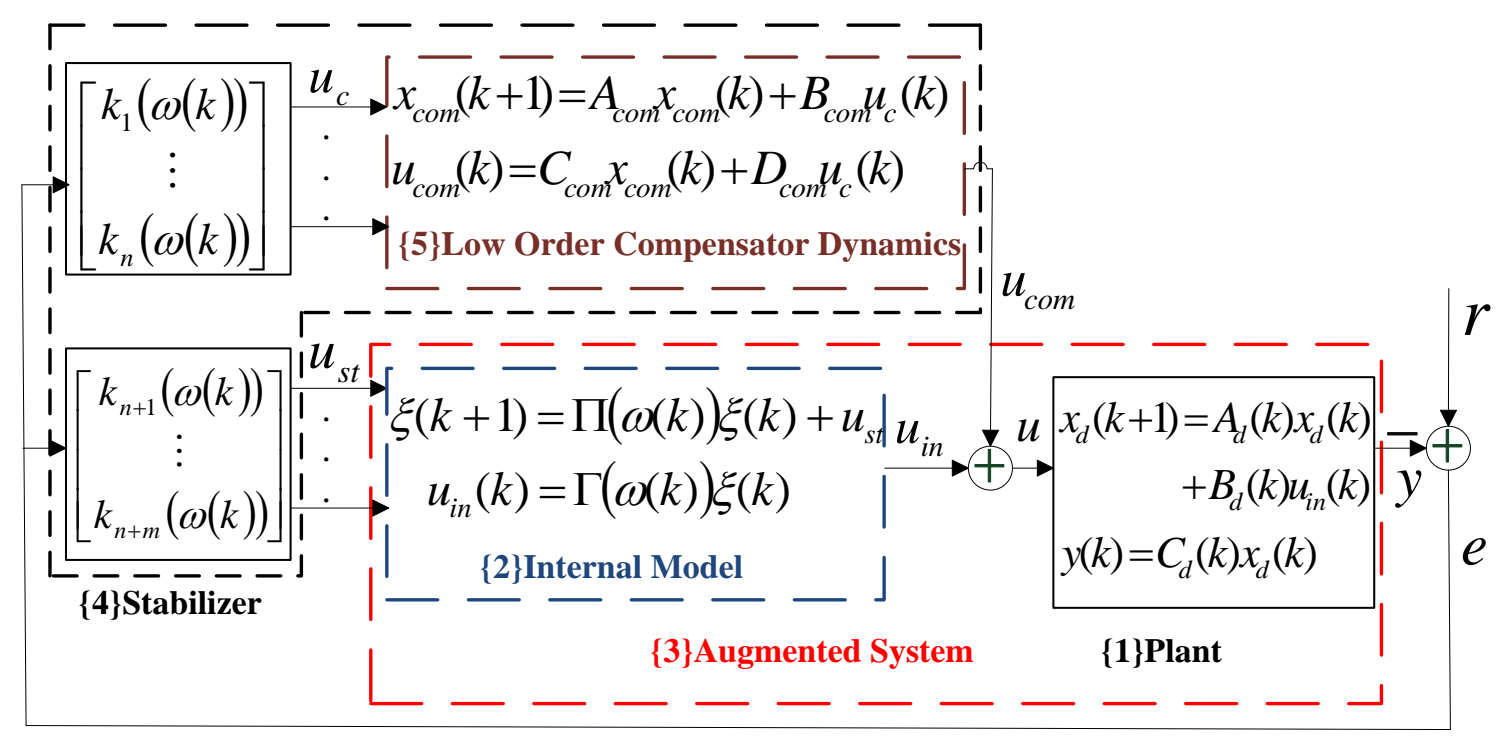

Figure 2.2. (b) Block diagram of the internal model based control system

\subsection{Control Design}

The control system block diagram is shown in Figure 2.2. The control design consists of two parts. First part is to have an angle varying internal model, which is determined by both the signal generating dynamics (Eq. (2.2)) and plant dynamics (Eq. (2.1)). The second part is to design a stabilizer that can stabilize the augmented system.

Following the method described in [13], the angle varying internal model can be designed as two interconnected subsystems as shown in Figure 2.2 (a). The internal model can be considered as the feed-forward control maintains the tracking error as zero. Due to the LTI generating dynamic (Eq. (2.2)), the internal model in the angle domain is independent of the reference profile, which provides the flexibility to track different reference profiles. Once the order of the generating dynamics is decided, the internal model is only dependent on the plant dynamics (Eq. (2.1)), which is usually fixed and of low order. Consider the state-space 
representation of the two interconnected subsystems of the internal model unit in Figure 2.2 (a) as

$$
\begin{aligned}
& \xi_{1}(k+1)=\Pi_{1}(\omega(k)) \xi_{1}(k)+\Psi_{1}(\omega(k)) u_{i n}(k) \\
& u_{r}(k)=\Gamma_{1}(\omega(k)) \xi_{1}(k) \\
& \xi_{2}(k+1)=\Pi_{2}(\omega(k)) \xi_{2}(k)-\Psi_{2}(\omega(k)) u(k) \\
& u_{\text {in }}(k)=\Gamma_{2}(\omega(k)) \xi_{2}(k)-D_{2}(\omega(k)) u(k)
\end{aligned}
$$

The designed internal model dynamics $\{2\}$ in Figure 2.2 (b) can be described as:

$$
\begin{aligned}
& \xi(k+1)=\Pi(\omega(k)) \xi(k) \\
& u_{\text {in }}(k)=\Gamma(\omega(k)) \xi(k)
\end{aligned}
$$

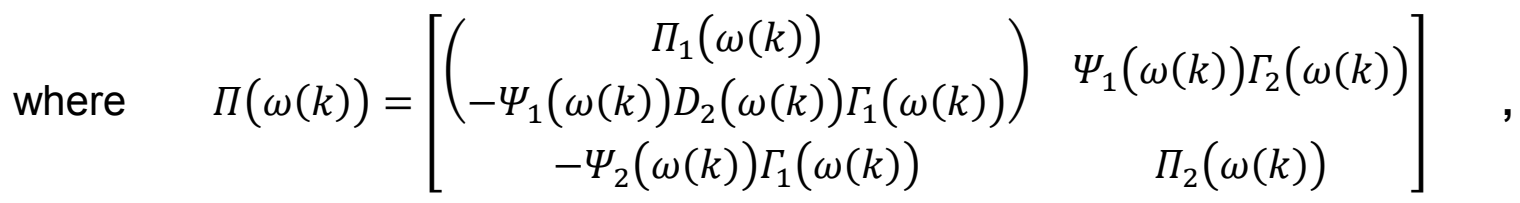
$\Gamma(\omega(k))=\left[\begin{array}{lll}-\Gamma_{1}(\omega(k)) D_{2}(\omega(k)) & \Gamma_{2}(\omega(k))\end{array}\right], \quad \xi(k)=\left[\begin{array}{ll}\xi_{1}(k) & \xi_{2}(k)\end{array}\right]^{T} \quad$ is the internal model state, and $u_{\text {in }}(k)$ is the control input signal from the internal model unit to the plant.

The stabilizer is designed based on the method proposed in [15]. It's essentially a parameter varying output injection based approach, where the stabilizer consists of a set of parameter varying gains and a low order compensator. This design structure enables a low order stabilization solution and thus avoids high computational cost. The stabilizer $\{4\}$ shown in Figure 2.2 (b) can be written as:

$$
u_{s t}(k)=K(\omega(k)) y(k)
$$

where $K(\omega(k))$ is the angle varying (speed dependent) control gain vector as (here we drop $\omega(k)$ for presentation clarity):

$$
K=\left[\begin{array}{llllll}
k_{1} & \cdots & k_{n} & k_{n+1} & \cdots & k_{n+m}
\end{array}\right]^{T}
$$

where $m=n+N-1, n$ is the order of the plant, $N$ is the order of the generating dynamics which is also the number of the sampling points. 
The closed loop system in Figure 2.2 (b) can be finally described as a linear parameter varying (LPV) system:

$$
\begin{aligned}
& \bar{X}_{c l p}(k+1)=\bar{A}_{c l p}(\omega(k)) \bar{X}_{c l p}(k) \\
& y(k)=\bar{C}_{a u g}(\omega(k)) \bar{X}_{c l p}(k)
\end{aligned}
$$

where $\quad \bar{A}_{\text {clp }}(\omega(k))=\bar{A}_{\text {aug }}(\omega(k))+\bar{B}_{\text {aug }}(\omega(k)) K(\omega(k)) \bar{C}_{\text {aug }}(\omega(k)) \quad$ and $\bar{X}_{c l p}(k)=\left[\begin{array}{lll}x_{d}(k) & \xi(k)\end{array}\right]^{T} . \bar{A}_{\text {aug }}(\omega(k)), \bar{B}_{\text {aug }}(\omega(k))$ and $\bar{C}_{\text {aug }}(\omega(k))$ are the coefficient matrices in the augmented system (the internal model + plant dynamics) $\{3\}$.

$$
\begin{aligned}
& \bar{A}_{\text {aug }}(\omega(k))=\left[\begin{array}{cc}
A_{d}(\omega(k)) & B_{d}(\omega(k)) \Gamma(\omega(k)) \\
0_{m \times n} & \Pi(\omega(k))
\end{array}\right]_{(n+m) \times(n+m)}, \\
& \bar{B}_{\text {aug }}(\omega(k))=\left[\begin{array}{ll}
0_{n \times n} & 0_{n \times m} \\
0_{m \times n} & I_{m \times m}
\end{array}\right]_{(n+m) \times(n+m)}, \\
& \bar{C}_{\text {aug }}(\omega(k))=\left[\begin{array}{ll}
C_{d}(\omega(k)) & 0_{1 \times m}
\end{array}\right]_{1 \times(n+m)} .
\end{aligned}
$$

\subsection{Convergence Regulation for Angle Varying Gain Based Stabilizer}

\section{Design}

While the system is exponentially stable, the state transition for the LPV system shown in (Eq. (2.3)) can be described as [20] :

$$
\left\|\bar{X}_{c l p}(k)\right\|^{2}<\frac{\alpha_{2}}{\alpha_{1}}\left\|\bar{X}_{c l p}(0)\right\|^{2}\left(1-\frac{c}{\alpha_{2}}\right)^{k}
$$

where $\alpha_{1}, \alpha_{2}$ and $\alpha_{2}>c>0$ are parameters determining the initial transient magnitude and convergence rate. This parameter should be embedded into the LMls for stabilizer synthesis so that the closed-loop transient performance can be controlled. 
The discrete Lyapunov function is defined as

$$
V(k)=\bar{X}_{c l p}^{T}(k) P_{k} \bar{X}_{c l p}(k)
$$

where $P_{k}$ is the Lyapunov matrix at current step $k$. The varying parameter (i.e. rotational speed $\omega$ ) space is divided into a series of grids as:

$$
\omega_{1}, \omega_{2}, \ldots \omega_{s}, \ldots \omega_{q}
$$

And the Lyapunov matrix and the stabilizer gain vector corresponding to each parameter grid can be denoted as:

$$
\begin{gathered}
P_{1}, P_{2}, \ldots P_{S}, \ldots P_{q} \\
K_{1}, K_{2}, \ldots K_{S}, \ldots K_{q}
\end{gathered}
$$

Once the control gains at each grid are calculated, the stabilizer gain value used at real-time control can be interpolated accordingly.

To enable exponential stability with desired transient (Eq. (4)), $V(k)$ and $P_{k}$ should satisfy:

$$
\begin{gathered}
\alpha_{1} I<P_{k}<\alpha_{2} I \\
V(k+1)-V(k)<-c\left\|\bar{X}_{c l p}(k)\right\|^{2}
\end{gathered}
$$

Eq. (2.5) could be easily represented as two LMI inequalities. To convert Eq. (2.6) into LMI form, re-writing Eq. (2.6) as:

$$
\bar{A}_{c l p}^{T}(\omega(k)) P_{k+1} \bar{A}_{c l p}(\omega(k))-\left(P_{k}-c I\right)<0
$$

Using the schur complement, one can have

$$
\left[\begin{array}{cc}
P_{k}-c I & \bar{A}_{c l p}^{T}(\omega(k)) P_{k+1} \\
P_{k+1} \bar{A}_{c l p}(\omega(k)) & P_{k+1}
\end{array}\right]>0
$$

where $P_{k+1}$ is the Lyapunov matrix at the discrete step $k+1$. Then the matrix inequality constraining the Lyapunov matrix at each grid point $\omega_{s}$ could be obtained by replacing $P_{k}$ in Eq. (2.7) with $P_{s}$ and also approximating $P_{k+1}$ as 


$$
\begin{aligned}
P_{k+1} & =P_{k}+\Delta \theta \frac{d P_{k}}{d \theta} \\
& =P_{S}+\Delta \theta \frac{d P_{s}}{d \omega_{s}} \frac{d \omega_{s}}{d \theta} \\
& \approx P_{S}+\Delta \theta \frac{P_{s+1}-P_{s}}{\omega_{s+1}^{i}-\omega_{s}^{i}} \frac{d \omega_{s}}{d \theta}=\Omega_{\mathrm{s}}
\end{aligned}
$$

With Eq. (2.8), Eq. (2.7) can be written as

$$
\left[\begin{array}{cc}
P_{s}-c I & \bar{A}_{c l p}^{T}\left(\omega_{s}\right) \Omega_{\mathrm{s}} \\
\Omega_{\mathrm{s}} \bar{A}_{c l p}\left(\omega_{s}\right) & \Omega_{\mathrm{s}}
\end{array}\right]>0
$$

By introducing a square matrix $F_{s}$, for $\Omega_{\mathrm{s}}>0$

$$
\left[\begin{array}{cc}
I & 0 \\
0 & F_{s} \Omega^{-1}{ }_{\mathrm{s}}
\end{array}\right]\left[\begin{array}{cc}
P_{s}-c I & \bar{A}_{c l p}^{T}\left(\omega_{s}\right) \Omega_{\mathrm{s}} \\
\Omega_{\mathrm{s}} \bar{A}_{c l p}\left(\omega_{s}\right) & \Omega_{\mathrm{s}}
\end{array}\right]\left[\begin{array}{cc}
I & 0 \\
0 & \Omega^{-1}{ }_{\mathrm{s}} F_{S}^{T}
\end{array}\right]>0
$$

Then, one has

$$
\left[\begin{array}{cc}
P_{s}-c I & \bar{A}_{c l p}^{T}\left(\omega_{s}\right) F_{s}^{T} \\
F_{S} \bar{A}_{c l p}\left(\omega_{s}\right) & F_{s}+F_{s}^{T}-\Omega_{\mathrm{s}}
\end{array}\right]>0
$$

where $\bar{A}_{c l p}\left(\omega_{s}\right)=\bar{A}_{a u g}\left(\omega_{s}\right)+\bar{B}_{a u g}\left(\omega_{s}\right) K_{s} \bar{C}_{a u g}\left(\omega_{s}\right)$.

Define $L_{s}=F_{s} K_{s}$ by removing the variable crossing term, Eq. (2.9) can be rewritten as

$$
\left[\begin{array}{cc}
P_{s}-c I & \left(F_{s} A_{\text {aug }}+F_{S} L_{s}\right)^{T} \\
F_{s} A_{\text {aug }}+F_{S} L_{s} & F_{S}+F_{s}^{T}-\Omega_{\mathrm{s}}
\end{array}\right]>0
$$

Then the control gain $K_{s}$ corresponding to the gridding point $\omega_{s}$ can then be obtained by $K_{s}=F_{S}^{-1} L_{s}$.

With Eq. (2.5) and Eq. (2.10) added to the LMls presented in [15], desired convergence rate and transient magnitude could be controlled by selecting proper value for parameters $c, \alpha_{1}$ and $\alpha_{2}$.

Note that the stabilizer obtained by LMIs (Eq. (2.5) and Eq. (2.10)) not only has the flexibility to adjust the convergence rate, but also has rotational 
accelerated speed robustness performance. This is very useful in practice due to commonly existence of time-varying accelerated speed. There is no need to maintain the angle acceleration $\frac{d \omega}{d \theta}$ as a constant. In the practical implementation, when the upper bound of the angle acceleration is known, the output feedback gain based stabilizer can be calculated offline. Then, the stabilizer can work for all cases below this upper bound. This result can be applied to the accelerated speed varying case when the upper bound is known.

\subsection{Simulation Results}

Consider the continuous-time transfer function of the plant mode used for the control design as

$$
\frac{Y(s)}{U(s)}=\frac{9.65 e 1 s+1.563 e 5}{s^{2}+4.931 e 2 s+1.33 e 5}
$$

Converting the plant dynamics into the discrete rotational angle domain in Eq. (2.1) with the parameters $A_{d}(k)=e^{A_{c} \Delta T_{k}}, B_{d}(k)=\left[e^{A_{c} \Delta T_{k}}-I\right] A_{c}^{-1} B_{c}, C_{d}(k)=C_{c}$, $\Delta T_{k}=(\Delta \theta / \omega(k)) \times(60 / 360)$, where $A_{c}=\left[\begin{array}{cc}-4.931 e 2 & -1.33 e 5 \\ 1 & 0\end{array}\right], \quad B_{c}=\left[\begin{array}{l}1 \\ 0\end{array}\right]$ and $C_{c}=[9.651 e 11.563 e 5], \Delta \theta=45^{\circ}, \omega(0)=600 \mathrm{rad} / \mathrm{min}, \dot{\omega}=24 \mathrm{rad} / \mathrm{min}$. From Eq. (2.1), the angle-varying $\mathrm{I} / \mathrm{O}$ representation of the plant are obtained as

$$
\begin{gathered}
y(k)=A^{-1} B[u(k)] \\
A=1+z^{-1} \alpha_{1}(k)+z^{-2} \alpha_{2}(k) \\
B=z^{-1} \beta_{1}(k)+z^{-2} \beta_{2}(k)
\end{gathered}
$$




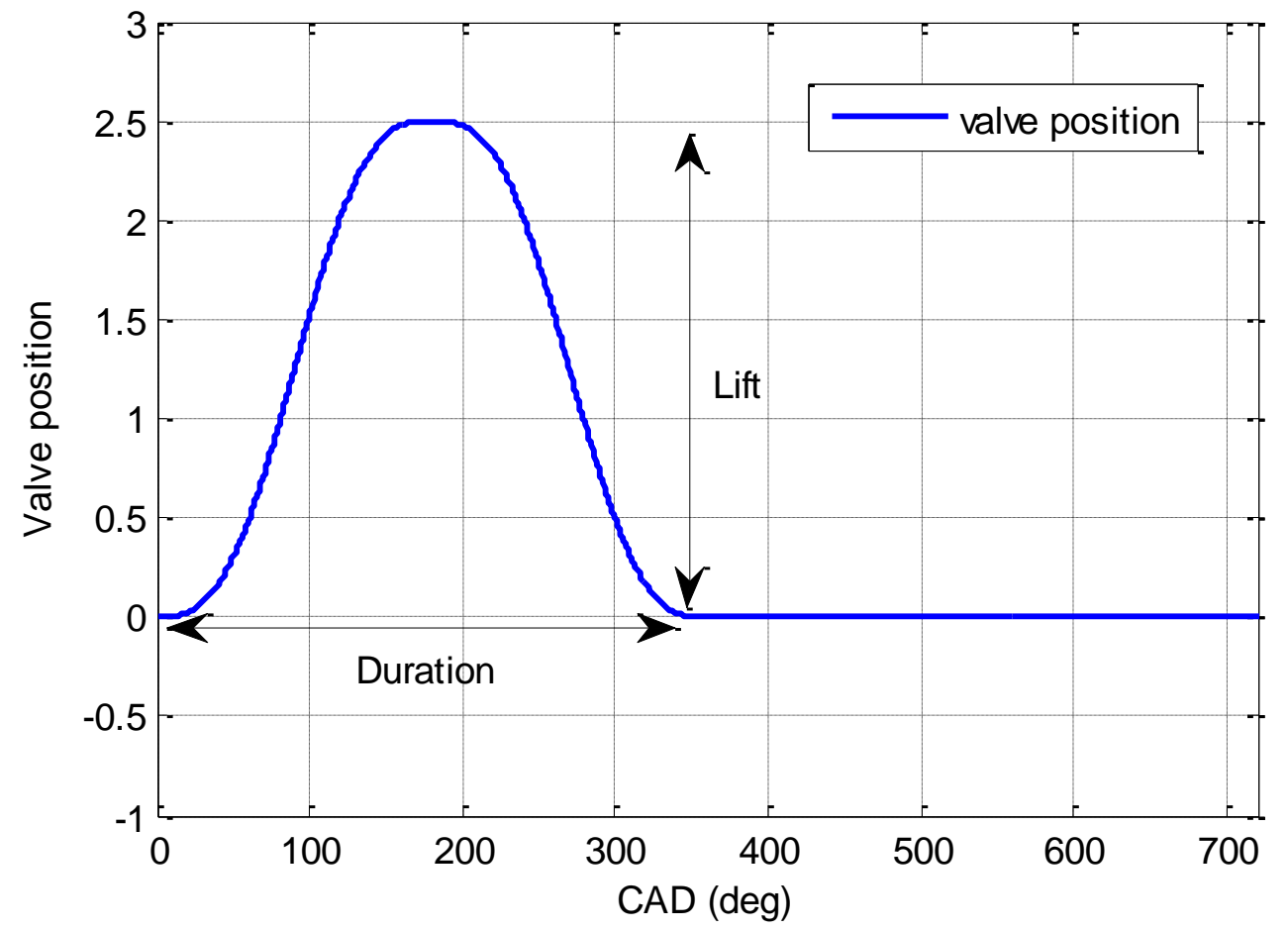

Figure 2.3. Reference profile.

16 sampling points are located to the desired engine valve motion reference profile shown in Figure 2.3. Then the angle-invariant generating dynamics is described as $r=\left(1-z^{-16}\right)^{-1}$.

The low-order compensator [11] whose dynamic are described in Figure 2.2 (b) is $1^{\text {st }}$ order dynamic with state parameter as

$$
\begin{aligned}
A_{\text {com }} & =\frac{-\beta_{2}(k)}{\beta_{1}(k+1)} \\
B_{\text {com }} & =\left[\begin{array}{ll}
\frac{-\beta_{2}(k)}{\beta_{1}(k+1) \beta_{1}(k)} & \frac{1}{\beta_{1}(k+1)}
\end{array}\right] \\
C_{\text {com }} & =1 \\
D_{\text {com }} & =\left[\begin{array}{ll}
\frac{-1}{\beta_{1}(k)} & 0
\end{array}\right]
\end{aligned}
$$


The varying parameter (i.e. rotational speed $\omega$ ) space is divided into 15 grids. And the angle varying (speed dependent) control gain matrix of the stabilizer is $K=\left[\begin{array}{lll}k_{1} & \cdots & k_{19}\end{array}\right]^{T}$ whose dimension is $19 \times 15$.
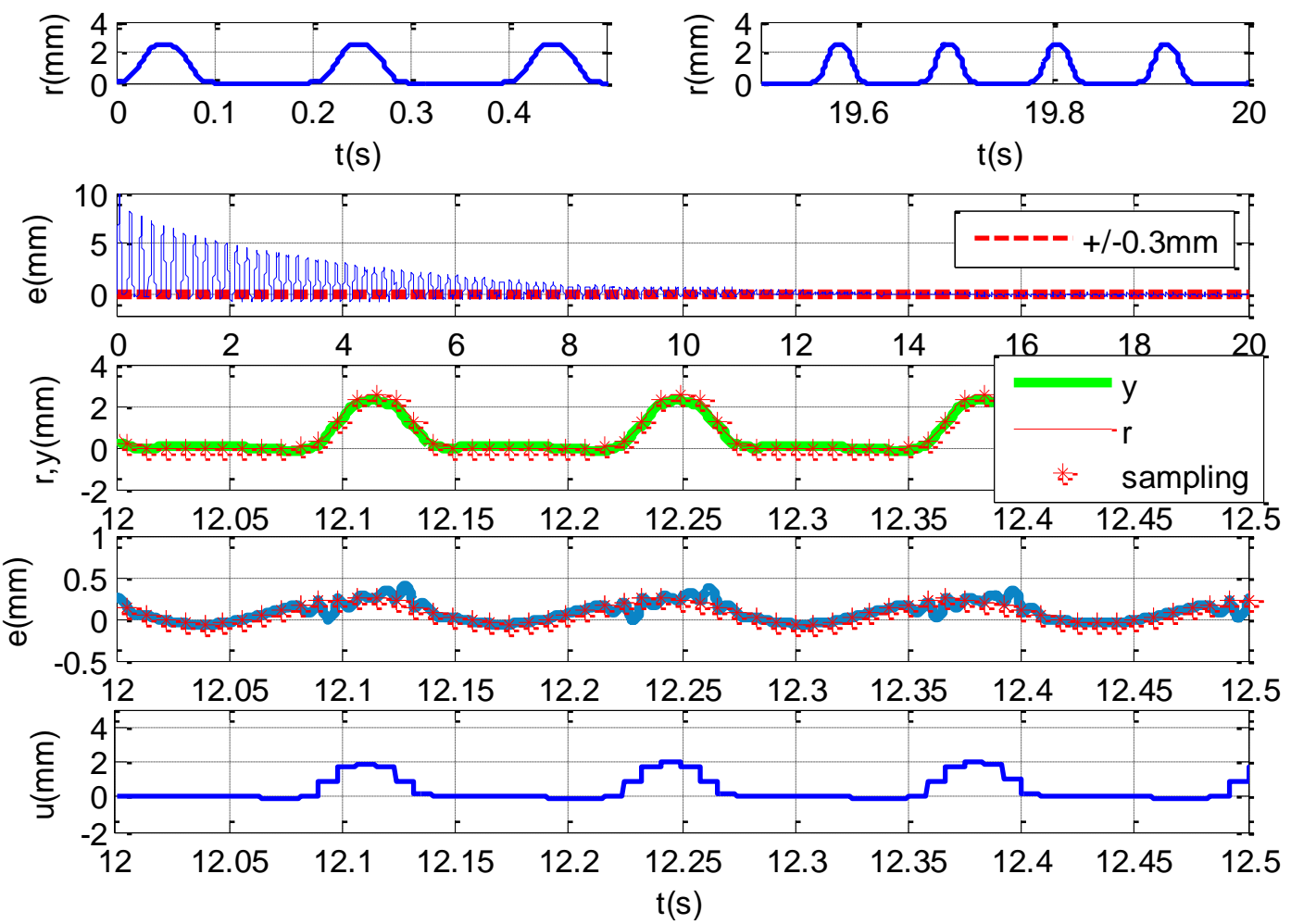

Figure 2.4. Simulational tracking results with 16-point sampling $[5-9 \mathrm{~Hz} @ 0.2 \mathrm{~Hz} / \mathrm{s}]$ with the origianl stabilizer [15]. 

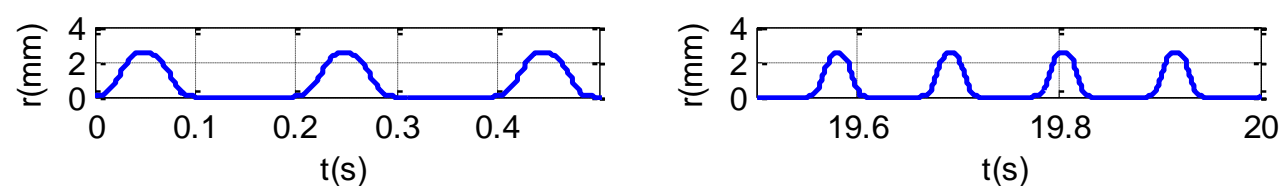

$\mathrm{t}(\mathrm{s})$
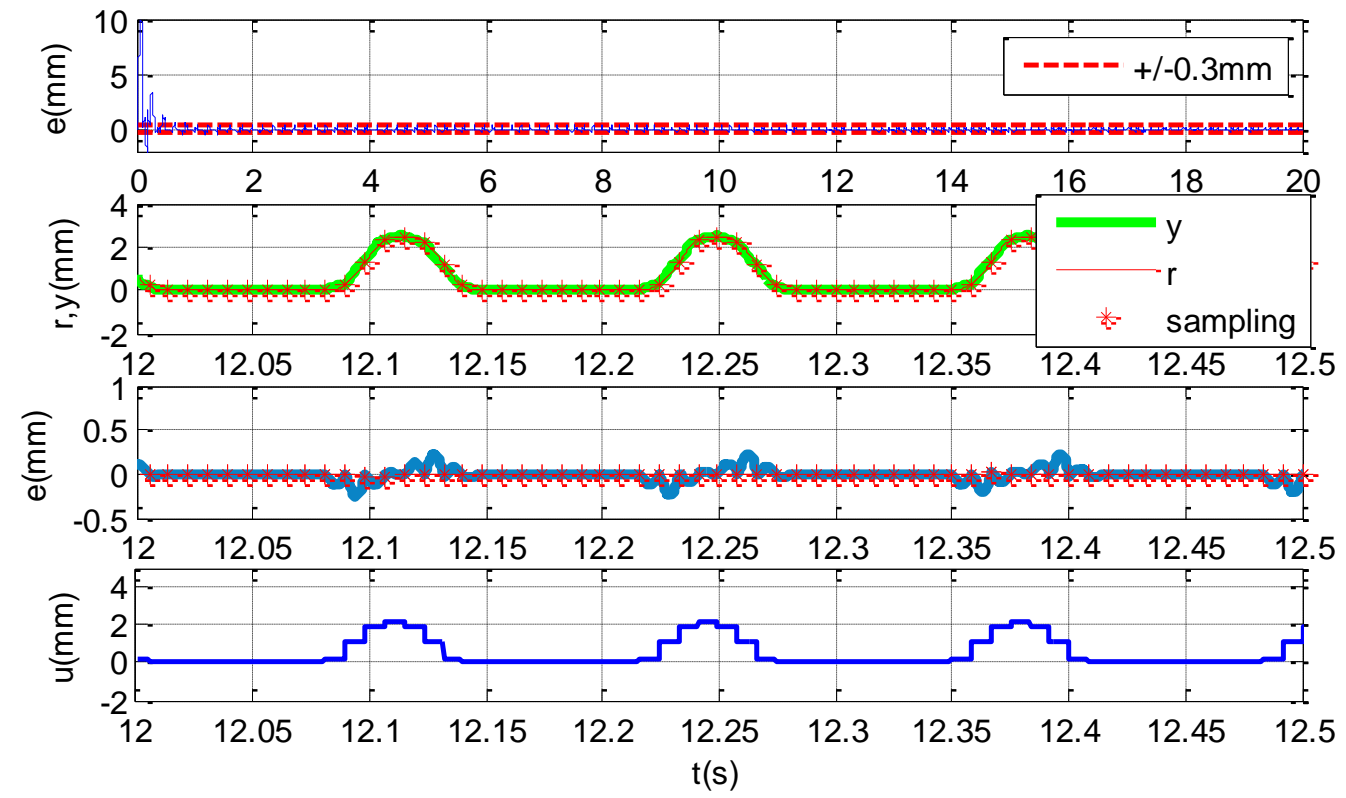

Figure 2.5. Simulational tracking results with 16-point sampling $[5-9 \mathrm{~Hz} @ 0.2 \mathrm{~Hz} / \mathrm{s}]$ with the convergence-improved stabilizer.

Figure 2.4 shows the simulation of the tracking performance by directly using the stabilizer presented in [15], and Figure 2.5 shows the simulation result with additional convergence constraints presented in this chapter for stabilizer synthesis. It is clearly seen that the tracking error converges to $+/-0.3 \mathrm{~mm}$ within $2 \mathrm{~s}$ in Figure 2.5, while the convergence time in Figure 2.4 is around $10 \mathrm{~s}$. This result clearly indicates the effectiveness of the convergence improvement presented in this chapter. Once reaching steady states, both controllers can maintain the tracking error level within a small interval as shown in the zoomed-in plots (the third, fourth, and fifth plots) in Figure 2.4 and Figure 2.5. 


\subsection{Conclusion}

This chapter investigates the reference tracking based on the internal model principle for a class of rotational angle dependent system. Motivated by the fact that the signal of interest is aperiodic in the time domain but periodic with respect to the rotational angle, the plant dynamics is converted into the angle domain and becomes angle-varying. In order to enable practical implementation, additional LMI constraints are added to the existing stabilizer synthesis approach to improve the control convergence rate, so that the initial tracking/disturbance rejection error can quickly converge to the steady state. The control approach is validated by simulations. 


\section{Chapter 3}

\section{Variable Sampling in the Angle Domain}

\subsection{Motivation}

It is found that fine sampling resolution of the reference profile can improve the tracking performance. One approach is to increase the number of sampling points per revolution in the angle domain, which is discussed in Chapter 2. However, more sampling points result in high order internal model and increase computational burden.

To solve this conflicting requirement of better tracking performance and less computation, instead of using a fixed sampling interval in Chapter 2, a varying interval sampling approach is presented to select the sampling location with varying intervals while maintaining the total number of the sampling points per revolution. However, varying sampling intervals will turn the plant (the valve actuator system) into a time-varying system. Fortunately this can be handled by the time-varying internal model based control [14].

An iterative approach for finding the optimal sampling intervals is proposed to select the sampling locations in the angle domain. The internal-model based control presented above is triggered only at the sampling points, but more tracking errors can be collected between these adjacent sampling points to guide the selection of the sampling locations with optimal sampling intervals. The iterative design is independent of the online internal model based feedback 
control loop. Note that this varying interval sampling design can be implemented to different profiles of interests.

\subsection{Variable Interval Sampling}

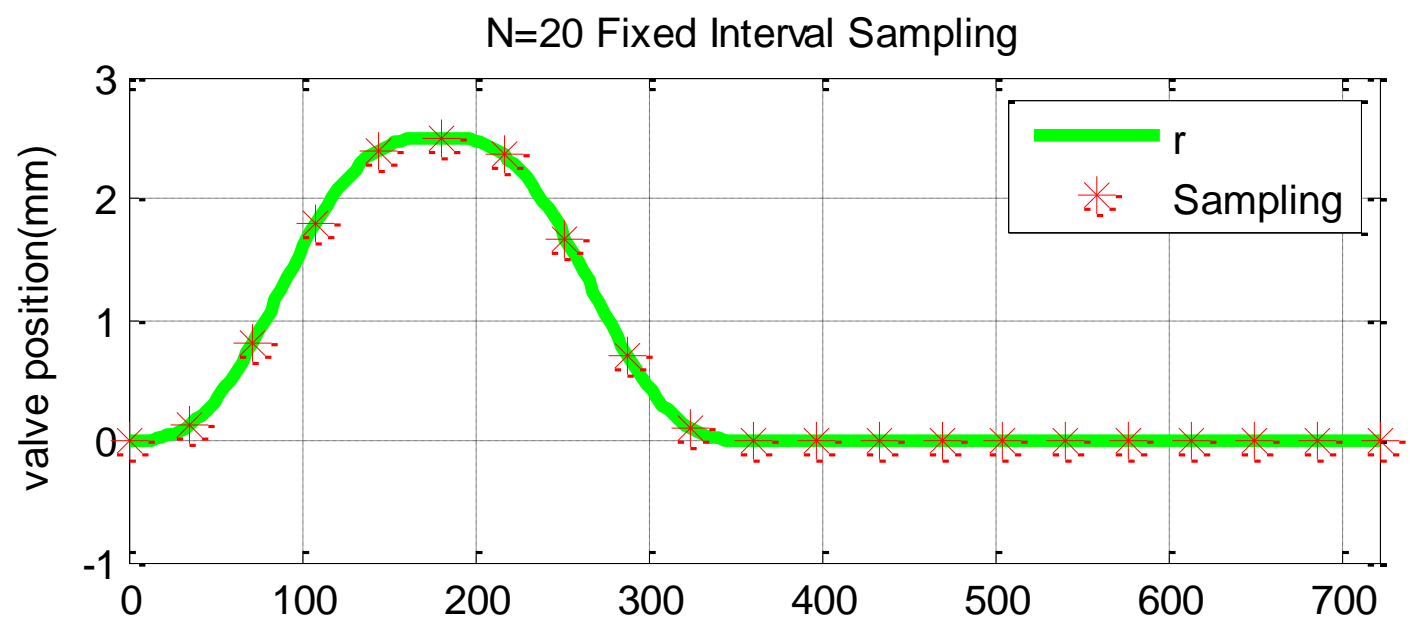

(a)

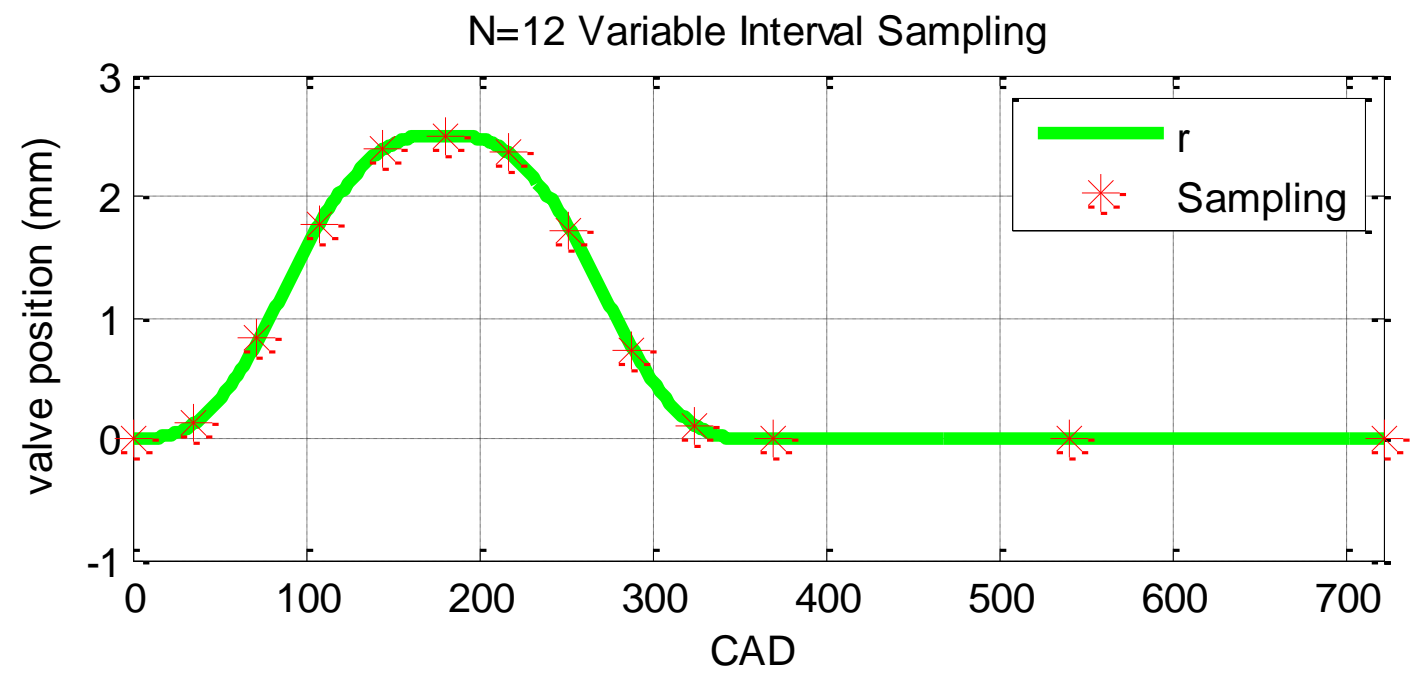

(b)

Figure 3.1. Comparison of fixed and variable interval sampling. 
To improve tracking performance, one typical approach is to increase the number of sampling points. For example, in order to obtain the resolution of $36^{\circ}$, the number of sampling points per revolution should be 20 as shown in Figure 3.1 (a). However, large number of sampling points per revolution can result in higher generating dynamics order $N$ (Eq. (2.2)) and therefore a high order internal model, which inevitably increases computational burden.

To address that, instead of using fixed angular sampling interval as shown in Figure 3.1 (a), one can use different sampling rate across in a rotational angle period based on the characteristics of a given reference/disturbance profile. As show in Figure 3.1 (b), the sampling resolution could be higher in the lift portion of the curve, and be lower at the flat portion. With this approach, the resolution of the lift portion can also reach $36^{\circ}$ by using only 12 samples total by distributing 10 samples in the lift portion and 2 points in the flat portion. This can significant reduce the number of sampling points per revolution.

\subsection{An Iterative Approach for Searching Optimal Sampling Interval}

Given the flexibility of varying sampling interval, determining the specific location for each sampling point is critical. For example, the sampling points may not be evenly distributed even inside the lift portion of the profile shown in Figure 3.1 (b). To determine the sampling pattern, an iterative approach for searching the optimal sampling intervals is proposed in this section.

The method proposed is to run the angle domain control in a number of iterations and update the varying sampling interval distribution pattern based on the tracking performance at the previous iteration as shown in Figure 3.2. Each iteration contains a number of revolution periods. 


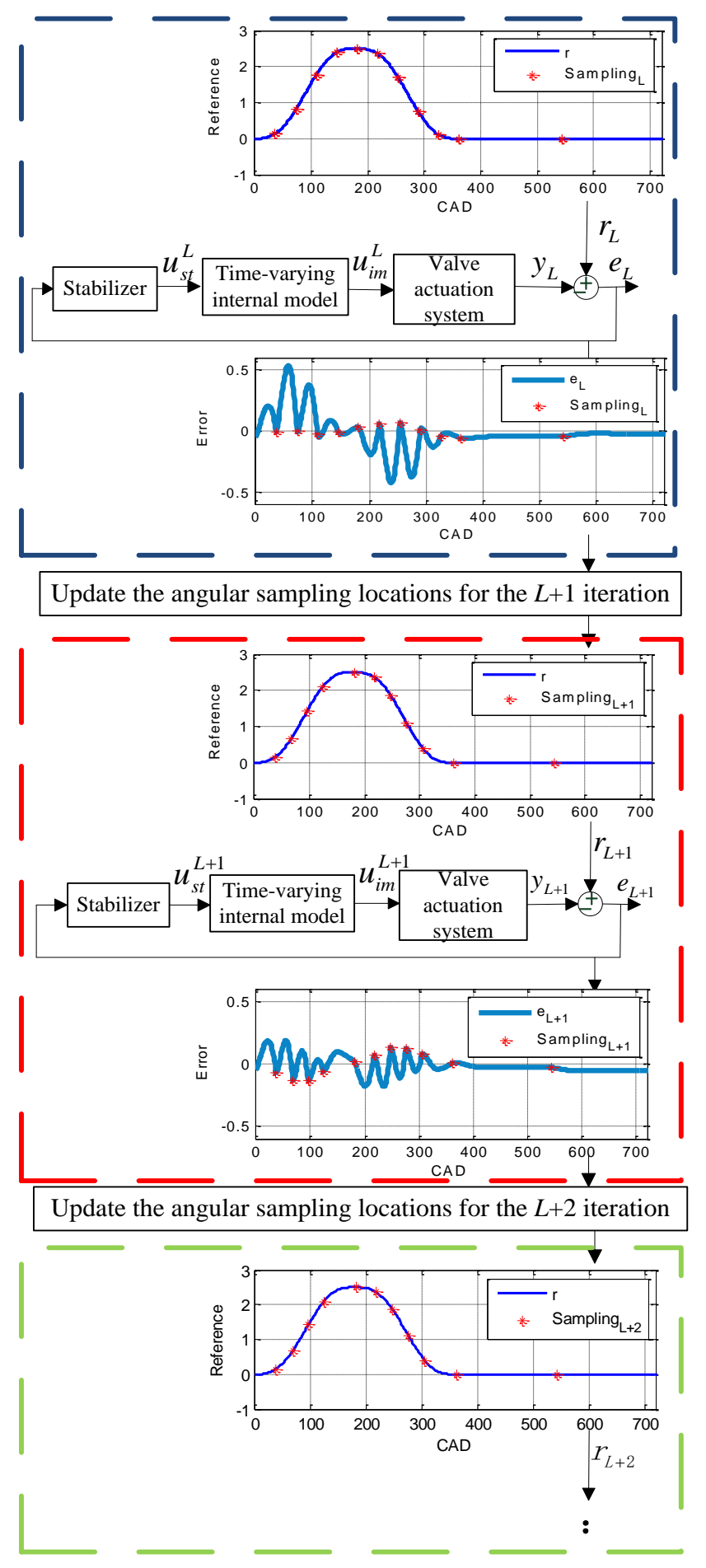

Figure 3.2. Block diagram of the iterative approach to search the optimal sampling interval for the time-varying internal model based camless engine valve actuation system. 
An initial sampling pattern is first given by choosing pre-determined the angular sampling locations as shown in Figure 3.3 (a). After running the angle based control for a few rotational periods until the tracking/rejection control reaches the steady state, the control error for one revolution period at the steady state could be obtained as shown in Figure 3.3 (b). It can be seen that error within certain sampling interval can be much higher than others and should be further reduced. The iterative sampling interval updating law proposed in this section will then update the sampling points locations as shown in Figure 3.2, and the process will be repeated until the peak error reaches a desirable level. .
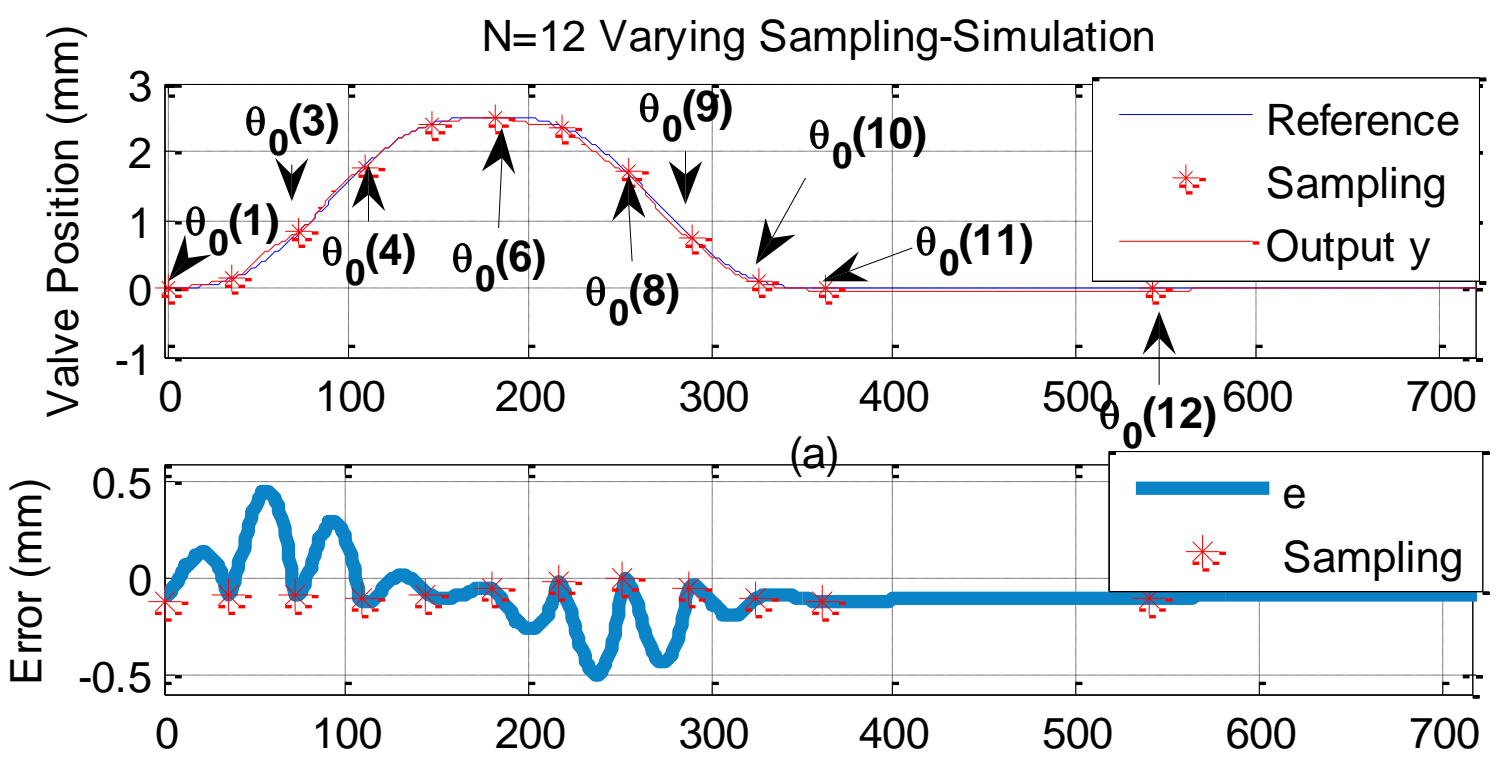

(b)

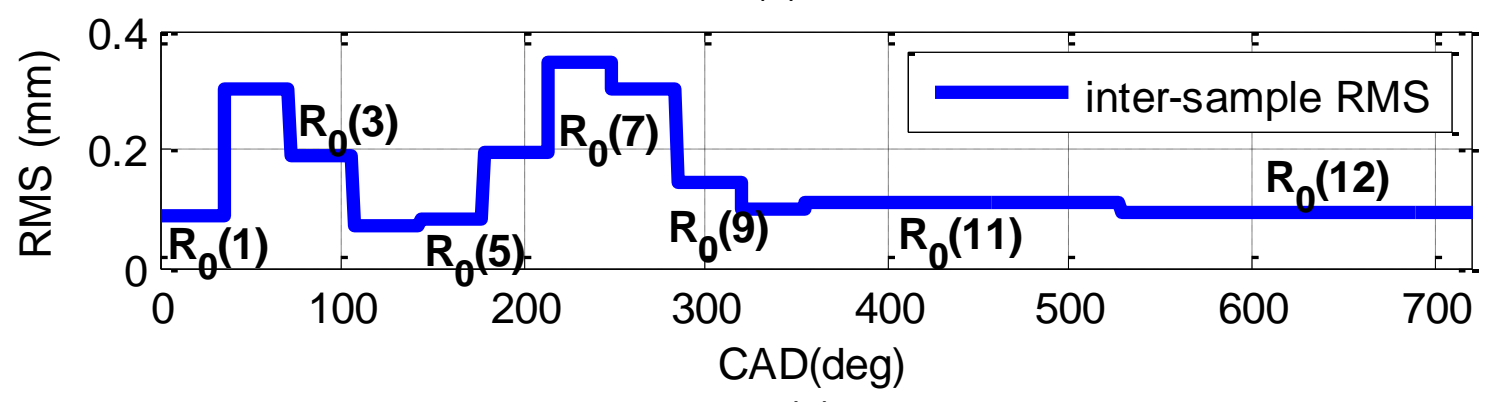

(c)

Figure 3.3. Initialize sampling intervals of the valve reference. 
The proposed design procedure can be summarized as follows:

Step 1). Initialize the sampling interval as shown in Figure 3.3 (a), where $\theta_{0}(1)$ to $\theta_{0}(N)$ represent the $1^{\text {st }}$ to the $N^{\text {th }}$ sampling point. More samplings are located at the critical tracking region such as the lift profile.

Step 2). With the sampling pattern initialized in step 1, run the tracking control until the tracking error converges to the steady state. For sampling interval searching purpose, the tracking error $e$ could be recorded with much higher measurement rate, and thus the error between the sampling points can be obtained as shown in Figure 3.3 (b). Then calculate the root mean square (RMS) value $R_{0}(i)$ of the tracking error $e$ between the sampling points $\theta_{0}(i)$ and $\theta_{0}(i+1), i=1, \cdots, N$ as shown in Figure 3.3 (c). If the RMS level for a particular sampling interval is too large, then the sampling interval should be shrunk to reduce the error.

Step 3) Update the angular sampling locations $\left[\theta_{L+1}(1), \cdots, \theta_{L+1}(N)\right]$ for the next iteration (here $L$ denotes the current iteration number). The update law can be written as:

$$
\begin{aligned}
& \theta_{L+1}(i)=\theta_{L}(i)+\widetilde{K}_{L} \times\left(R_{L}(i)-R_{L}(i-1)\right), \\
& i=2, \cdots, N, \quad L=0,1,2, \cdots
\end{aligned}
$$

where $\theta_{L+1}(i)$ and $\theta_{L}(i)$ are the $i^{\text {th }}$ sampling point for the $L+1$ and $L$ iterations respectively. $R_{L}(i)$ is the RMS value of the tracking error at the sampling interval $i$ for the current iteration $L . \widetilde{K}_{L}$ is the proportional gain. $\widetilde{K}_{L}$ is chosen as a constant to adjust the change of sampling angle, which can also be time varying if necessary.

Step 4) With the updated sampling intervals, run the angle domain tracking control again, and obtain the RMS value of the tracking error $R_{L+1}(i), \quad i=$ $1, \cdots, N$.

Step 5) Define $\tilde{E}_{L+1}(i)=R_{L+1}(i)-R_{L+1}(i-1)$, which is the difference between the RMS error of the adjacent sampling intervals $i-1$ and $i$. Select a 
dead zone $D Z$. If $D Z>\left|\widetilde{E}_{L+1}(i)\right|$ for all sampling points $i=2, \cdots, N$, then stop searching. Otherwise, the algorithm should go back to Step 3.

Step 6) The final sampling pattern can therefore be obtained once the iterative process stops. The overall flow of the sampling interval searching could be described as Figure 3.4.

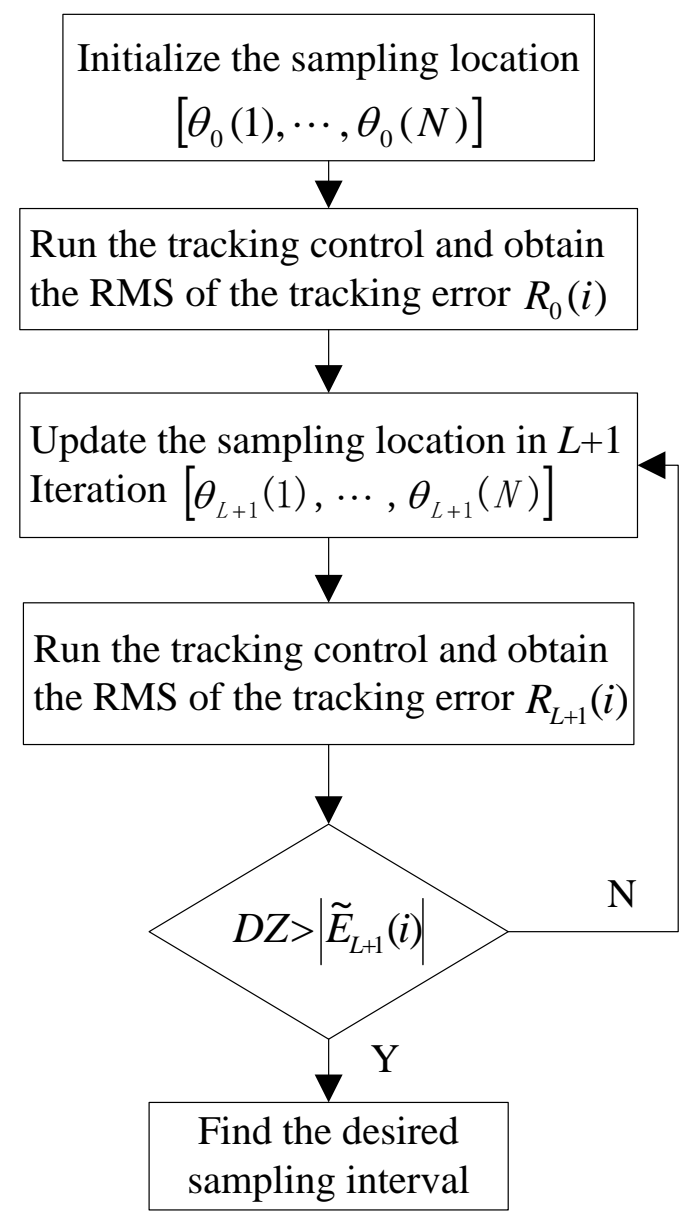

Figure 3.4. Flow chart of the iterative approach to search the desired sampling interval.

To avoid non-convergence in the sampling interval searching, the gain $\widetilde{K}_{L}$ in the searching algorithm should not be too large. The proper value for $\widetilde{K}_{L}$ could be tuning in practice. It is also possible to use simulation to offline pre-select the $\widetilde{K}_{L}$ 
value by minimizing the RMS value of the tracking error $e$ in the next iteration before conducting experiments. In addition, a reasonable searching stopping threshold $D Z$ is also important. The tracking error may not be effectively minimized if $D Z$ is too large, while a too small $D Z$ could result in nonconvergence of the searching process.

\subsection{Experiment Results}

The experimental results with the proposed varying interval sampling design are investigated on a time-varying internal model based camless engine valve actuation system. The camless engine valve actuation system will be described in details in Chapter 4. In this chapter, 12-ponit fixed and variable sampling results are compared to validate the iterative approach to search the desired sampling interval.
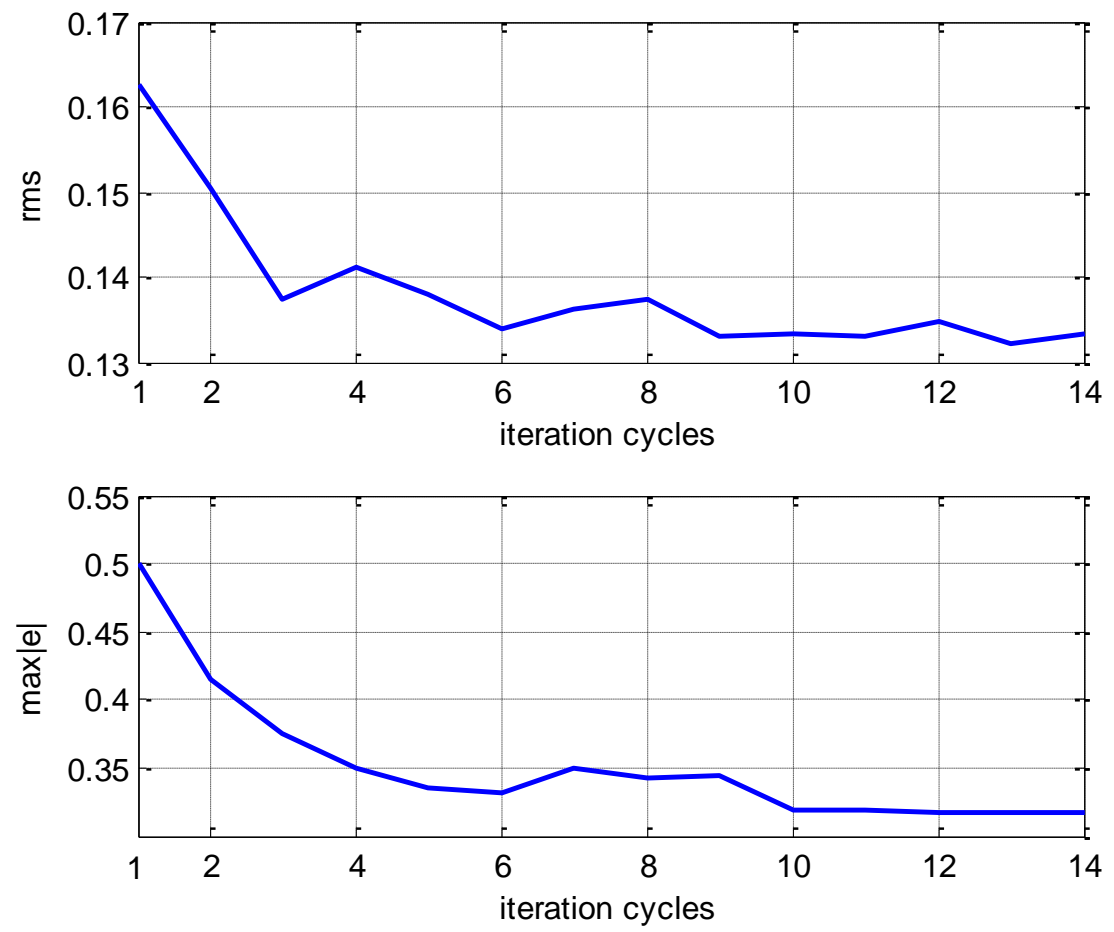

Figure 3.5. Convergence of the varying interval sampling design with $\widetilde{K}_{L}=20$. 
Figure 3.5 shows the convergence result with $\widetilde{K}_{L}=20$ and the dead zone $D Z$ as $4 \times 10^{-2} \mathrm{~mm}$ in the varying interval sampling design to find the desired variable interval sampling with internal model unit order $N=12$. The maximum peak value of the tracking error converge to $0.3166 \mathrm{~mm}$ after 14 cycles, meanwhile, RMS value of the tracking error decreases.
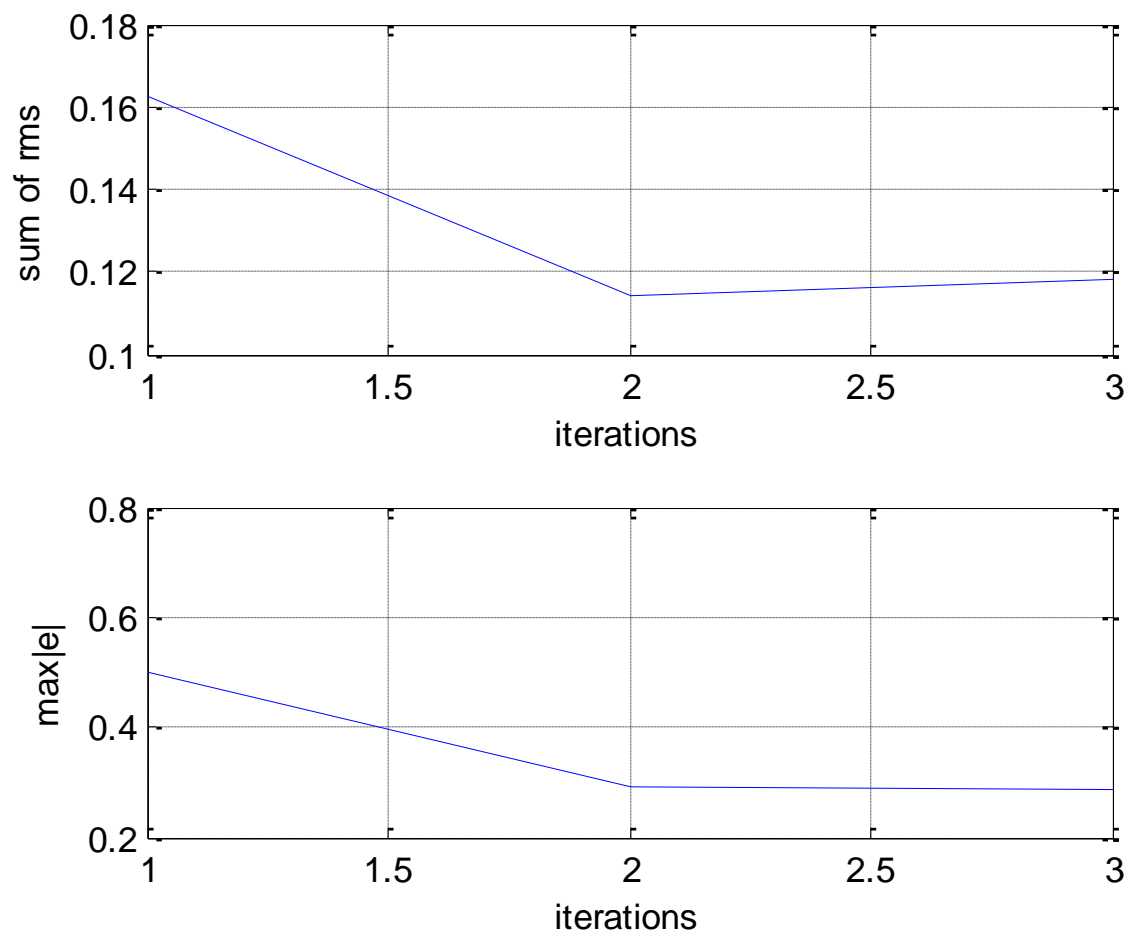

Figure 3.6. Convergence of the iterative varying interval sampling searching with $\left[\widetilde{K}_{1}\right.$,

$$
\left.\widetilde{K}_{2}, \widetilde{K}_{3}\right]=[28,22,20] .
$$

In each iteration cycle, the camless engine valve actuation system is run to obtain the steady states of the tracking error. Thus, it runs the valve system 14 times to obtain Figure 3.5, which is time-consuming. In order to save time and improve the convergence, simulation results can be used to offline choose a better $\widetilde{K}_{L}$ in the next iteration before conducting experiments. Figure 3.6 shows the convergence with the searching gain $\left[\widetilde{K}_{1}, \widetilde{K}_{2}, \widetilde{K}_{3}\right]=[28,22,20]$ and same dead zone $D Z$ as $4 \times 10^{-2} \mathrm{~mm}$. After 3 cycles, the algorithm converges as shown 
in Figure 3.6 which is much faster than one in Figure 3.5. Both RMS value and maximum peak value of the tracking error in Figure 3.6 are smaller than the numbers in Figure 3.5.

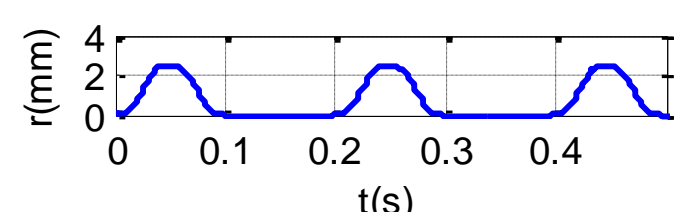

$\mathrm{t}(\mathrm{s})$

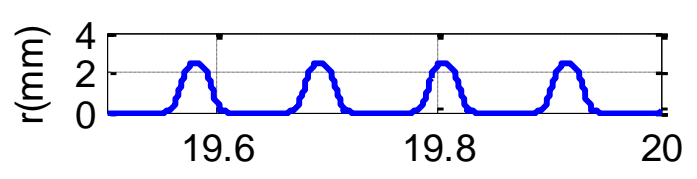

$\mathrm{t}(\mathrm{s})$

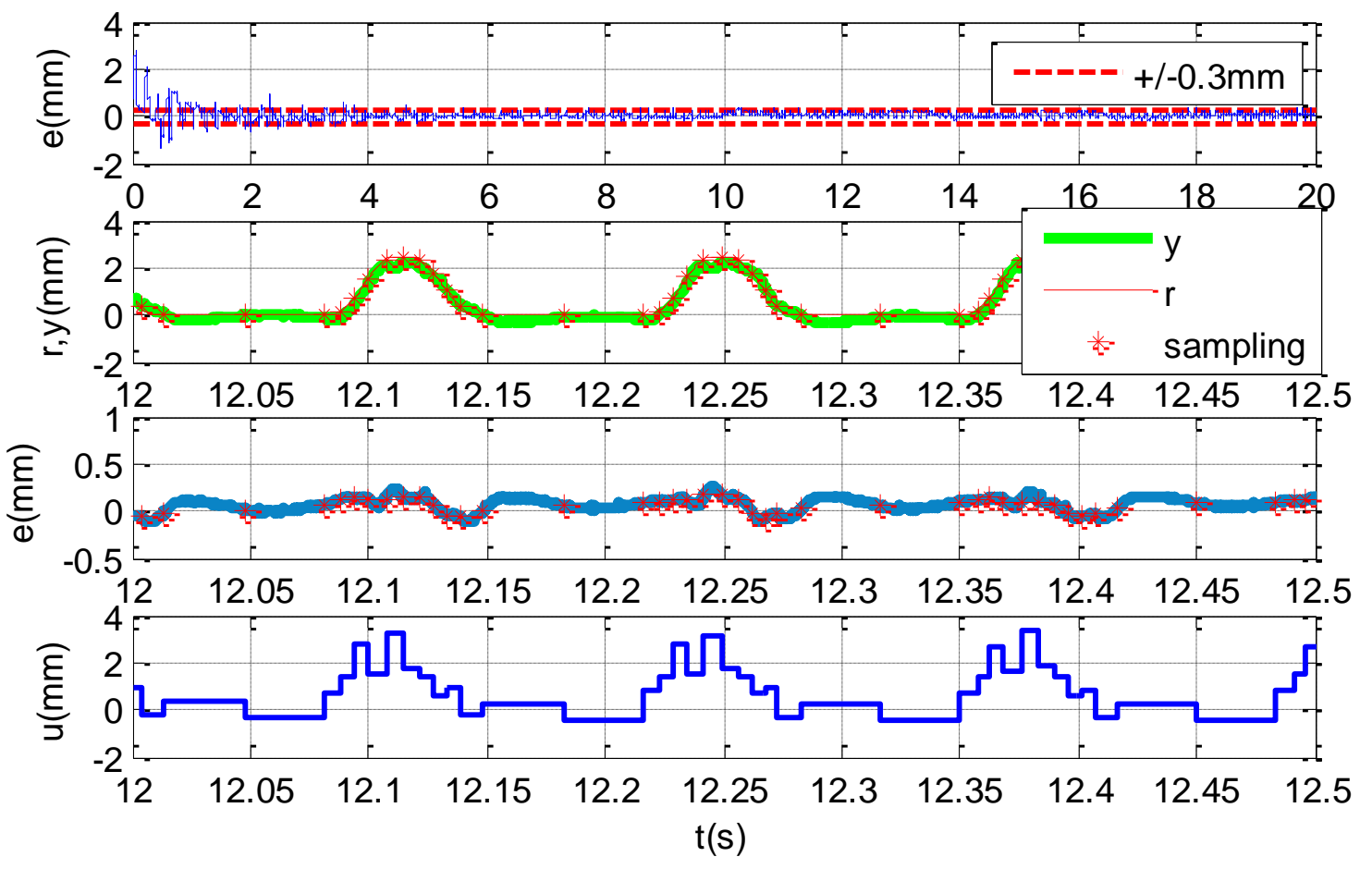

Figure 3.7. Experimental tracking results with 12-point variable interval sampling $[5-9 \mathrm{~Hz}$ @ $0.2 \mathrm{~Hz} / \mathrm{s}]$ with the convergence-improved stabilizer.

For the reference profile $(2.5 \mathrm{~mm}$ lift and 360 CAD duration shown in Figure 2.3), the initial variable angular sampling pattern $\theta_{0}$ is pre-selected as $\left[0^{\circ}, 36^{\circ}\right.$, $72^{\circ}, 108^{\circ}, 144^{\circ}, 180^{\circ}, 216^{\circ}, 252^{\circ}, 288^{\circ}, 324^{\circ}, 360^{\circ}, 540^{\circ}$ ], with the resolution $\Delta \theta=360 /(N-2)=36^{\circ},(N=12)$ between $0-360^{\circ}$, and $\Delta \theta=(720-360) / 2=$ $180^{\circ}$ between $360^{\circ}-720^{\circ}$. The algorithm presented in Chapter 3.3 is then used to 
search the optimal sampling interval with the searching gain $\left[\widetilde{K}_{1}, \widetilde{K}_{2}, \widetilde{K}_{3}\right]=[28$, $22,20]$ and the dead zone $D Z$ as $4 \times 10^{-2} \mathrm{~mm}$. After 3 cycles, the algorithm converges as shown in Figure 3.6, and the final sampling pattern $\theta_{\text {final }}$ is obtained as: $\left[0^{\circ}, 40.2267^{\circ}, 69.6115^{\circ}, 101.8124^{\circ}, 143.9833^{\circ}, 180.0000^{\circ}\right.$, $217.2356^{\circ}, 246.5425^{\circ}, 273.8535^{\circ}, 302.7529^{\circ}, 360.0000^{\circ}, 540.0000^{\circ}$ ].

The tracking performance using the optimal sampling interval is shown in Figure 3.7. It can be observed that the tracking error at steady state is much less than that shown in Figure 3.8, where the fixed interval sampling is used.

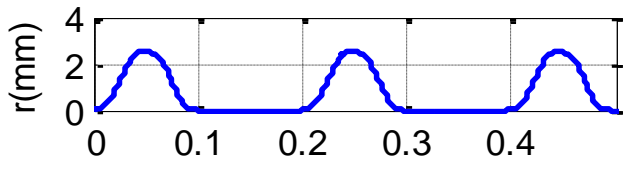

$\mathrm{t}(\mathrm{s})$

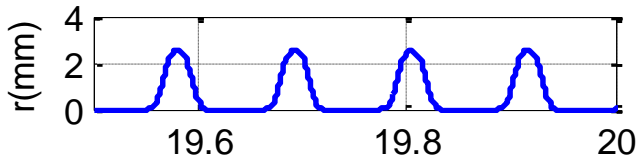

$\mathrm{t}(\mathrm{s})$
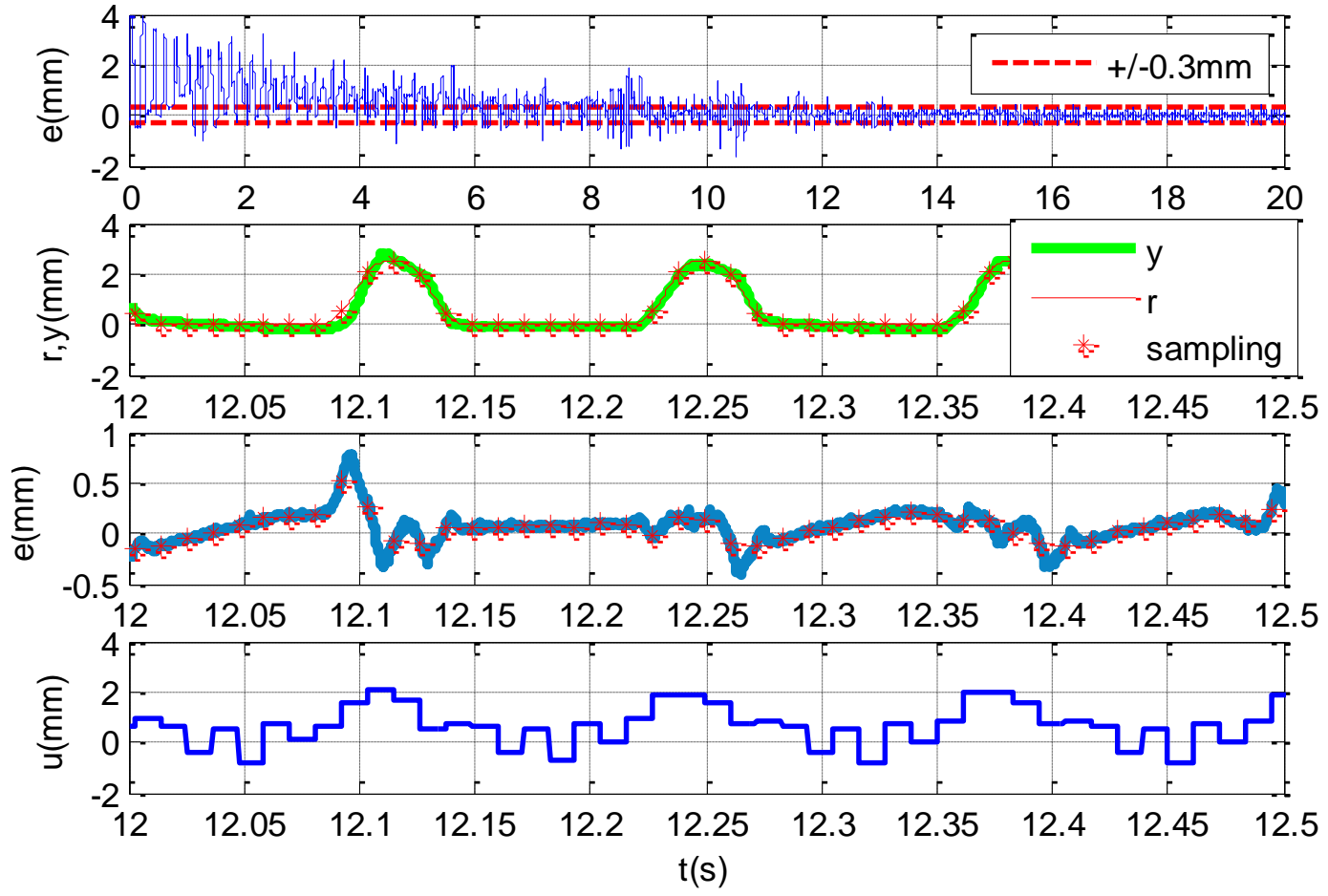

Figure 3.8. Experimental tracking results with 12-point fixed interval sampling $[5-9 \mathrm{~Hz} @$ $0.2 \mathrm{~Hz} / \mathrm{s}]$ with the original stabilizer. 


\subsection{Conclusion}

This chapter investigates the variable interval sampling based time-varying tracking control in the rotational angle domain. It is found that more sampling points per revolution provide better tracking performance but increase the computational burden. To solve the problem, a varying interval sampling approach is presented to optimize the angular sampling interval for the reference profile, while maintaining the same total number of sampling points. The tracking performance is improved by considering the tracking errors between the sampling points in selecting the optimal sampling intervals. Experimental results from a time-varying internal model based camless engine valve actuation system 12-point fixed and variable interval sampling demonstrate the effectiveness of the proposed method. 


\section{Chapter 4}

\section{Experimental Investigations}

To validate the proposed angle based control, it is applied to a fully flexible valve actuation system. The fully flexible valve actuation (FFVA) or "camless" engine uses electronically based actuators to replace the mechanical connection to the camshaft for the engine valve actuation. This design decouples the engine valve from the crankshaft in order to allow flexibility of the valve lift, duration, and timing over a wide operation range, which can enable significant improvement in fuel economy, emissions [17-18], and power output. However, without the mechanical connection to the crankshaft, precise control of the engine valve motion is critical to avoid possible collision between the piston and the engine valve, and also to ensure accurate intake/exhaust air control.

\subsection{Camless Engine Valve Hardware setup}

The camless valve actuation system used for the experiment is shown in Figure 4.1. The system consists of a hydraulic actuator, a spool valve, a voice coil motor, a hydraulic pump, a non-contact displacement sensor (Microstrain NC-DVRT 1.0), and an engine poppet valve assembly. The displacement of the tapped poppet valve stem is measured by Microstrain NC-DVRT 1.0 with $10 \mathrm{KHz}$ bandwidth. The spool position is controlled by the voice coil motor (BEI Kimco Magnetics: LA13-12-000A) with a sensitivity of $K_{F}=9.79 \mathrm{~N} / \mathrm{A}$. The voice coil 
current is generated by a high-bandwidth power amplifier (Advanced Motion Controls: 12A8).

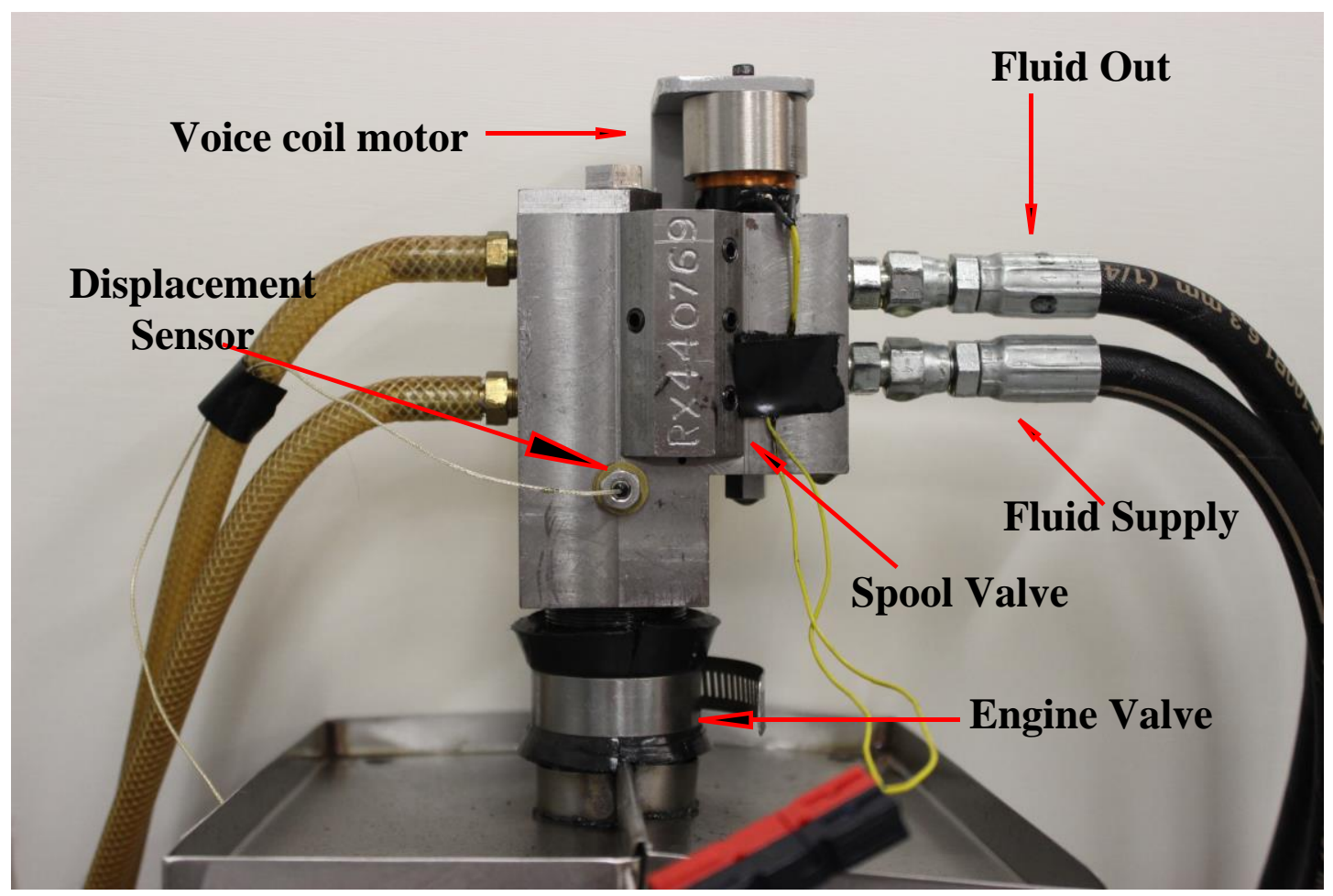

Figure 4.1. Camless engine valve actuation system setup.

In experiments, the engine valve position is measured and feed back to the angle domain controller, which then controls the voice coil motor/actuator for engine valve position control as shown in Figure 4.2. The coil motor controls the movements of the 3-way spool valve to vary the flow of the high pressure fluid from the pump into and out of the hydraulic piston. The high pressure fluid above the hydraulic piston provides the force against the spring which is loaded to the engine valve, and forces the engine valve down/up. A proportional controller is first used to stabilizer the boundary stable system. Based on the difference of the 
actual valve position $y$ and the control command $u$ to the spool valve, this proportional controller controls the current $i$ in the voice coil motor.

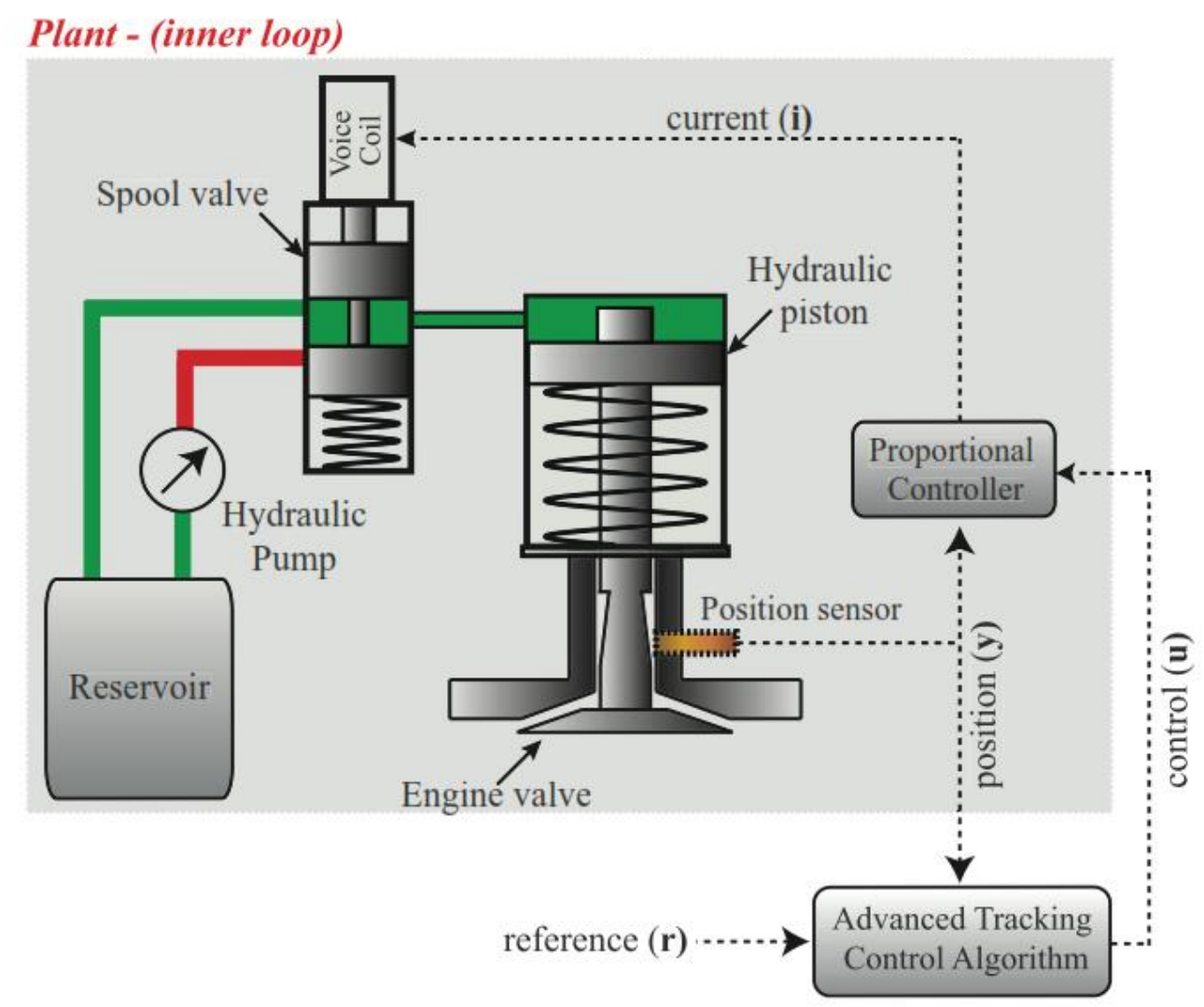

Figure 4.2. Schematic of camless engine valve actuation system.

The desired engine valve motion reference profile is shown in Figure 2.3, which consists of a lift portion (for air flowing in/out) and a valve closing portion. 


\subsection{Experiment Validation for Convergence-improved Stabilizer}

Simulation results in Chapter 2 show the effectiveness of the convergenceimproved stabilizer. In this Chapter, experimental tracking results with different numbers of sampling points further validate its effectiveness of convergenceimproved stabilizer. The results with the original stabilizer in [15] are used for comparison analysis.

\subsubsection{2-point fixed interval sampling case}

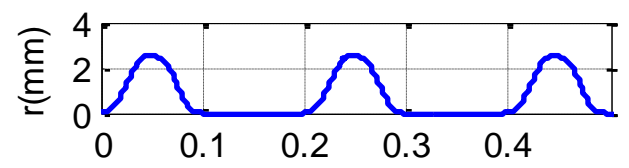

$\mathrm{t}(\mathrm{s})$
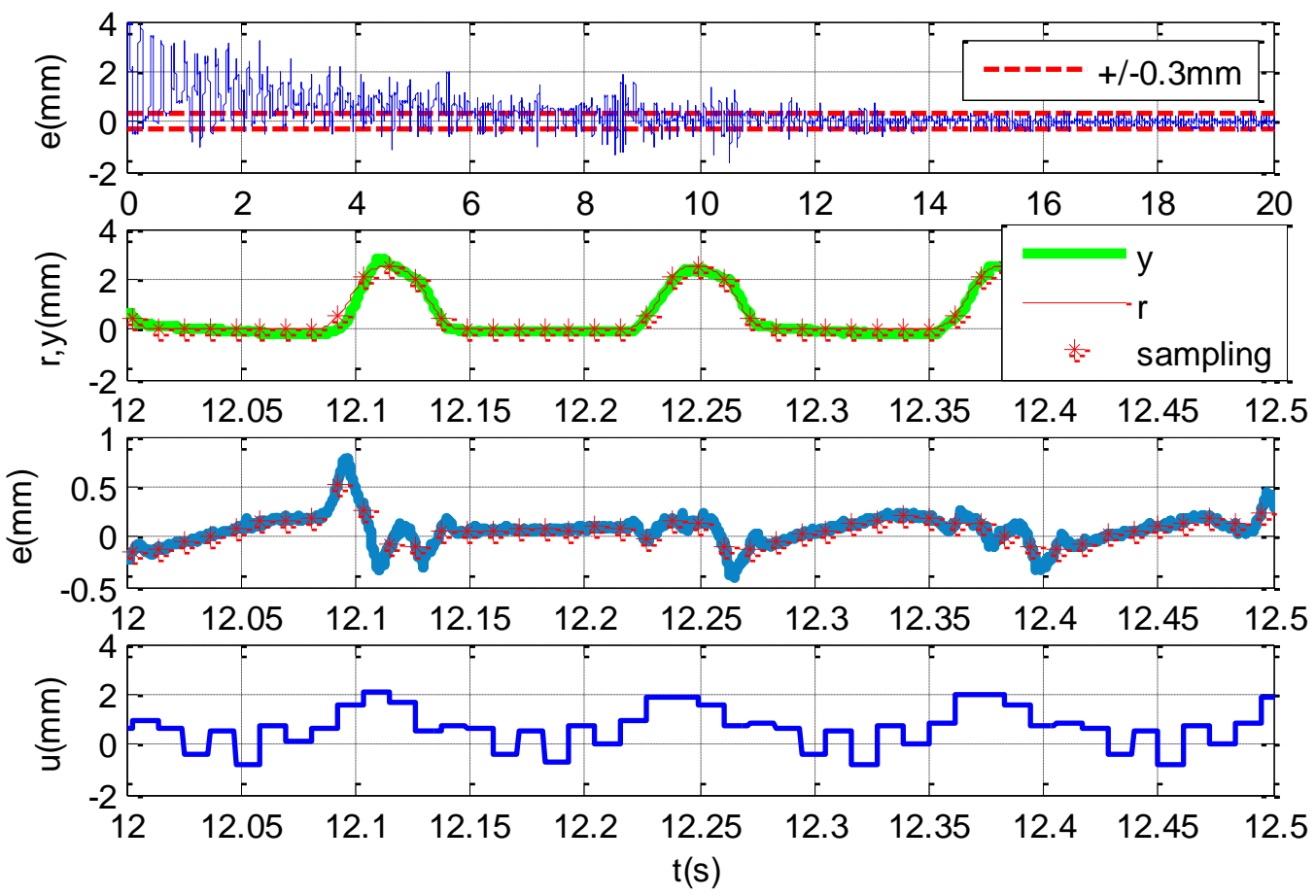

Figure 4.3. Experimental tracking results with 12-point fixed interval sampling $[5-9 \mathrm{~Hz} @$ $0.2 \mathrm{~Hz} / \mathrm{s}]$ with the origianl stabilizer. 

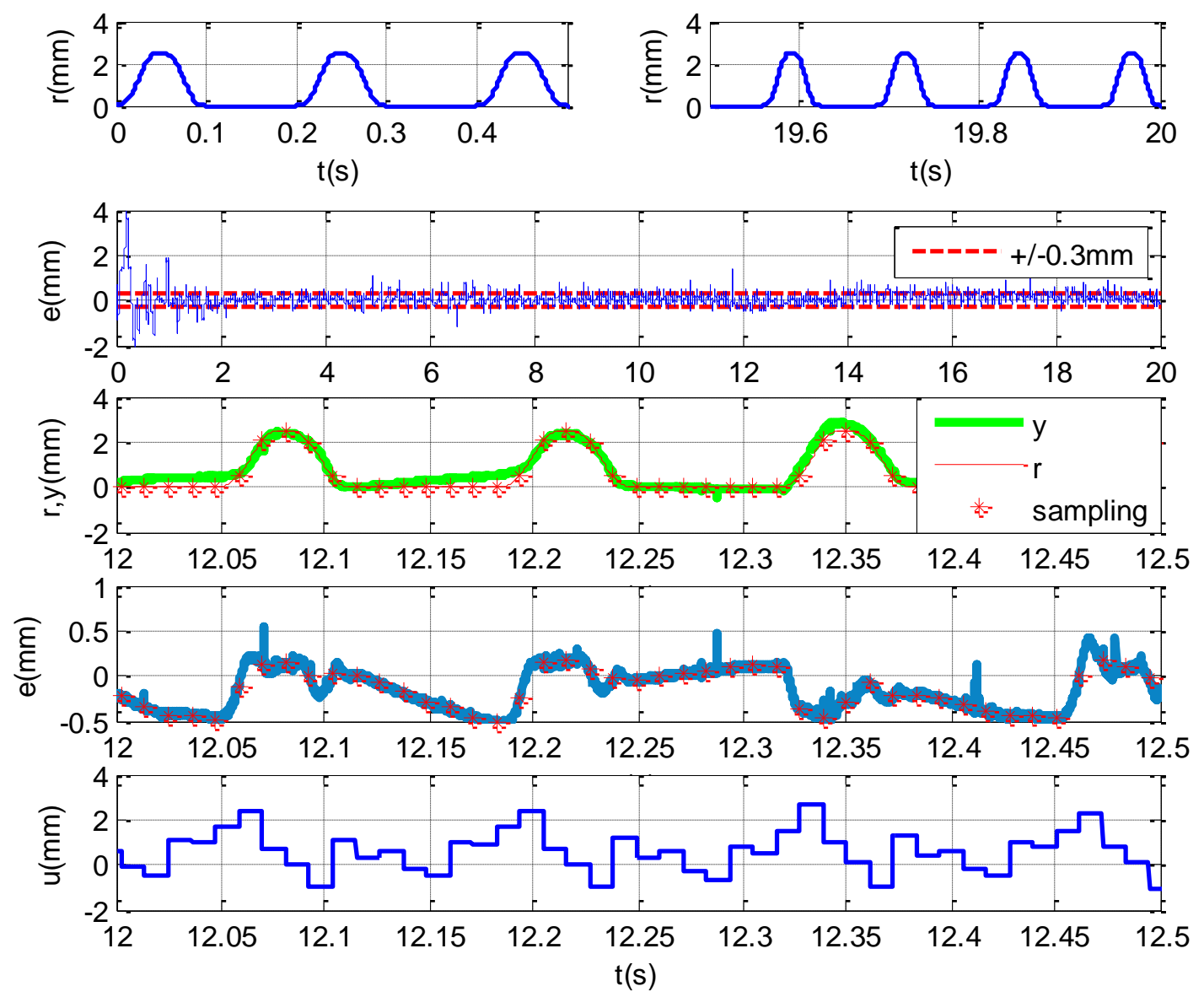

Figure 4.4. Experimental tracking results with 12-point fixed interval sampling $[5-9 \mathrm{~Hz} @$ $0.2 \mathrm{~Hz} / \mathrm{s}]$ with the convergence-improved stabilizer.

Figure 4.3 shows the tracking performance by directly using the stabilizer presented in [15], and Figure 4.4 shows the results with additional convergence constraints presented in Chapter 2 for stabilizer synthesis. Both of them are tested with a $12^{\text {th }}$ order internal model based system with fixed interval angular samplings. It is clearly seen that the tracking error converges to $+/-0.3 \mathrm{~mm}$ within $2 \mathrm{~s}$ in Figure 4.4, while the convergence time in Figure 4.3 is around $12 \mathrm{~s}$. Also, the peak transient magnitude is about $4 \mathrm{~mm}$ in Figure 4.4 comparing with $5 \mathrm{~mm}$ 
shown in Figure 4.3. These results clearly indicate the effectiveness of the convergence improvement by the convergence-improved stabilizer presented in Chapter 2. Once reaching steady states, both controllers can maintain the tracking error level within a small interval as shown in the zoomed-in plots (the third, fourth, and fifth plots) in Figures 4.3 and 4.4.

\subsubsection{4-point fixed interval sampling case}

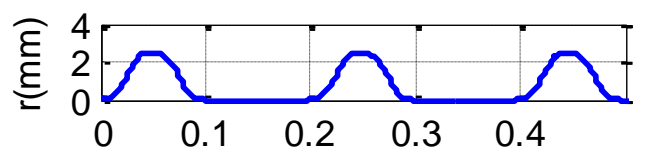

$\mathrm{t}(\mathrm{s})$

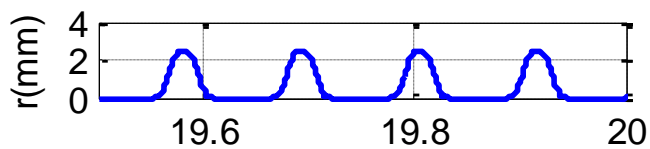

$\mathrm{t}(\mathrm{s})$
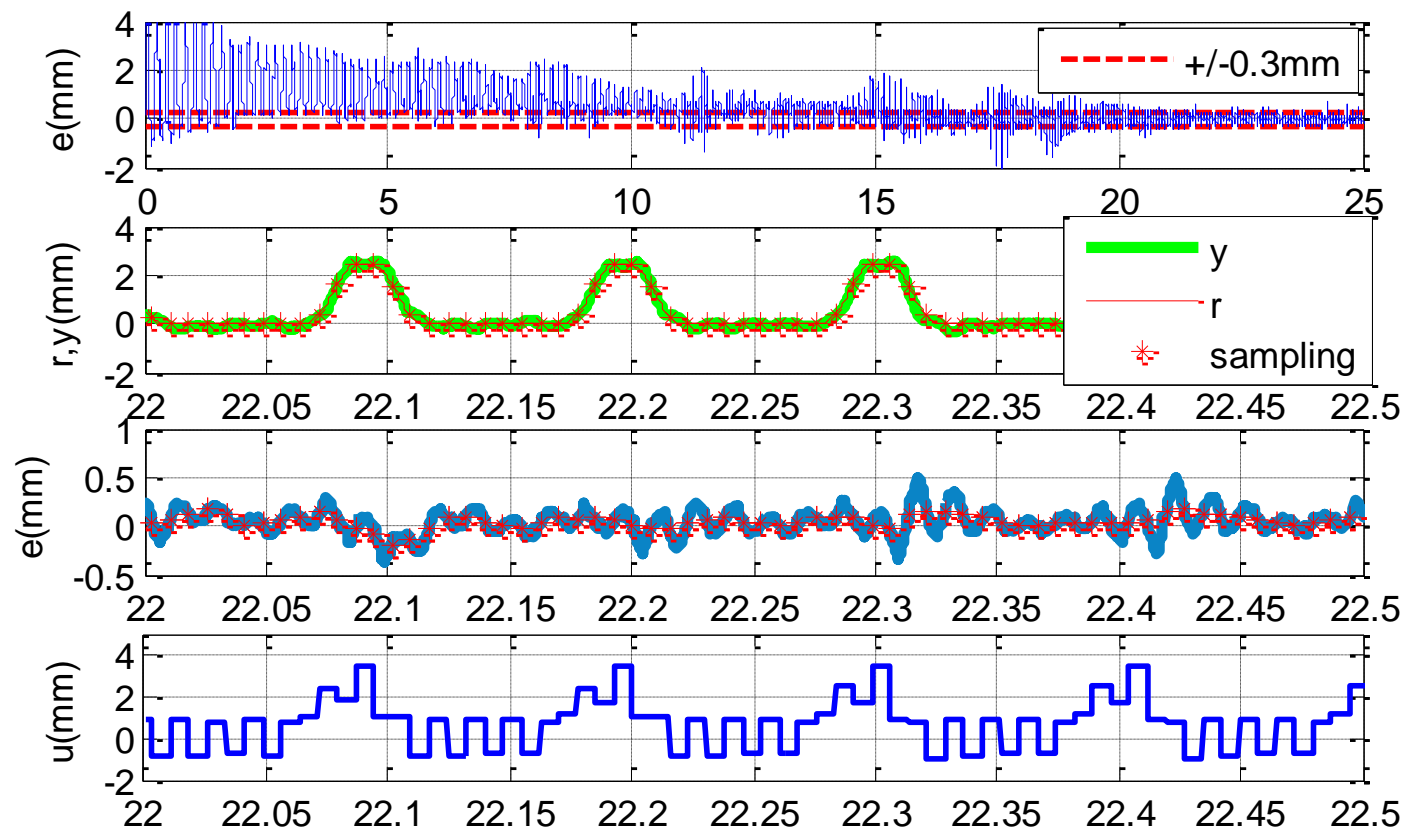

$\mathrm{t}(\mathrm{s})$

Figure 4.5. Experimental tracking results with 14-point fixed interval sampling $[5-9 \mathrm{~Hz} @$ $0.2 \mathrm{~Hz} / \mathrm{s}]$ with the origianl stabilizer. 


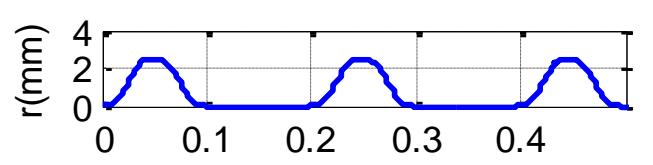

$\mathrm{t}(\mathrm{s})$

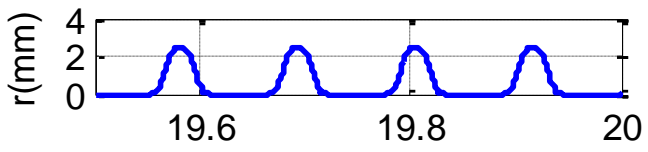

$\mathrm{t}(\mathrm{s})$
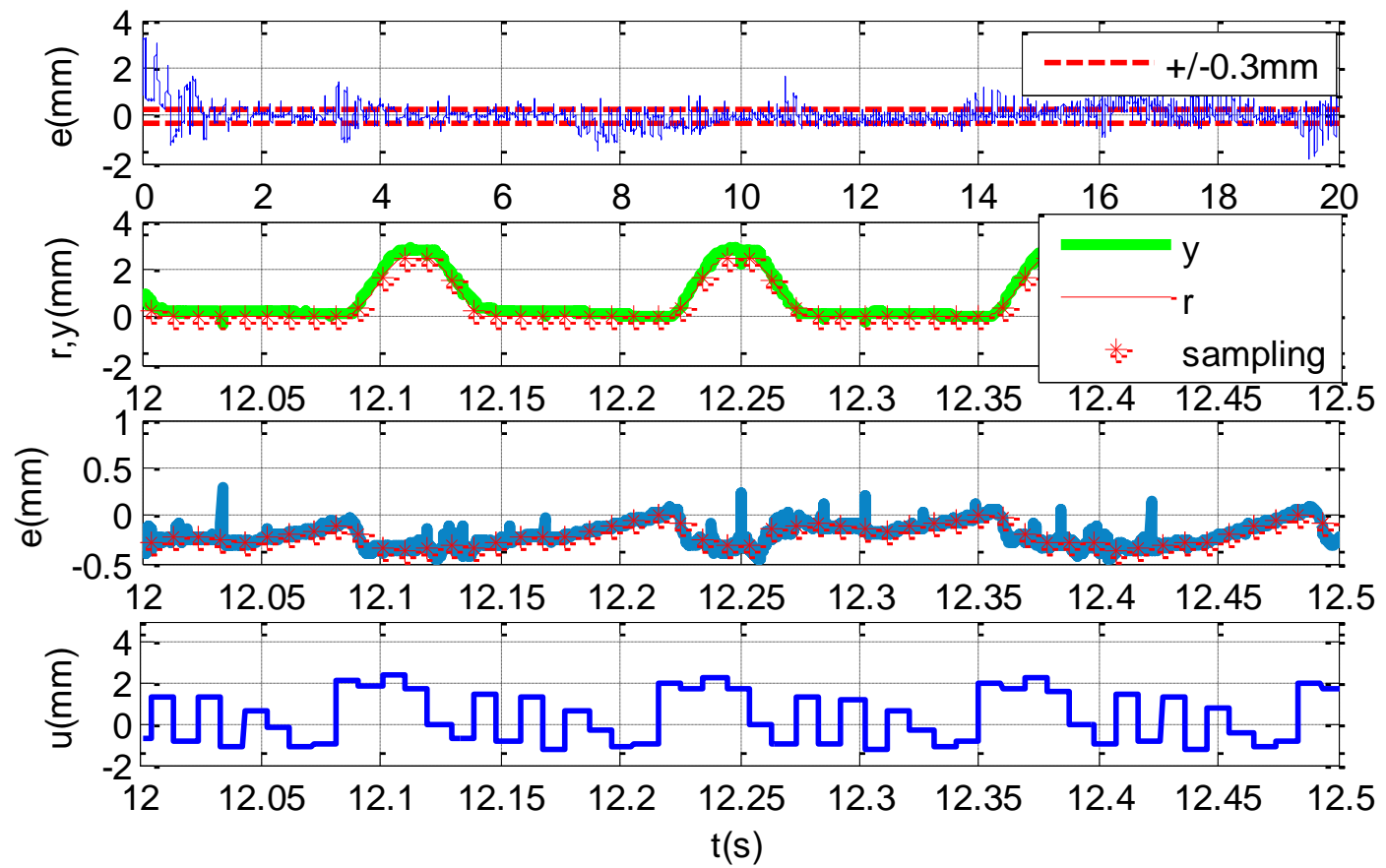

Figure 4.6. Experimental tracking results with 14-point fixed interval sampling $[5-9 \mathrm{~Hz} @$ $0.2 \mathrm{~Hz} / \mathrm{s}]$ with the convergence-improved stabilizer.

This convergence improvement can be obviously seen for larger number of the sampling points per revolution which is corresponding to the higher order of the internal model based system. Experimental tracking results wih 14-point sampling are shown in Figure 4.5 and Figure 4.6 with fixed and variable intervals respectively. The tracking error in Figure 4.6 converges to $+/-0.3 \mathrm{~mm}$ also within $2 \mathrm{~s}$, which is much faster than the convergence time (20.4s) in Figure 4.5. 


\subsubsection{6-point fixed interval sampling case}
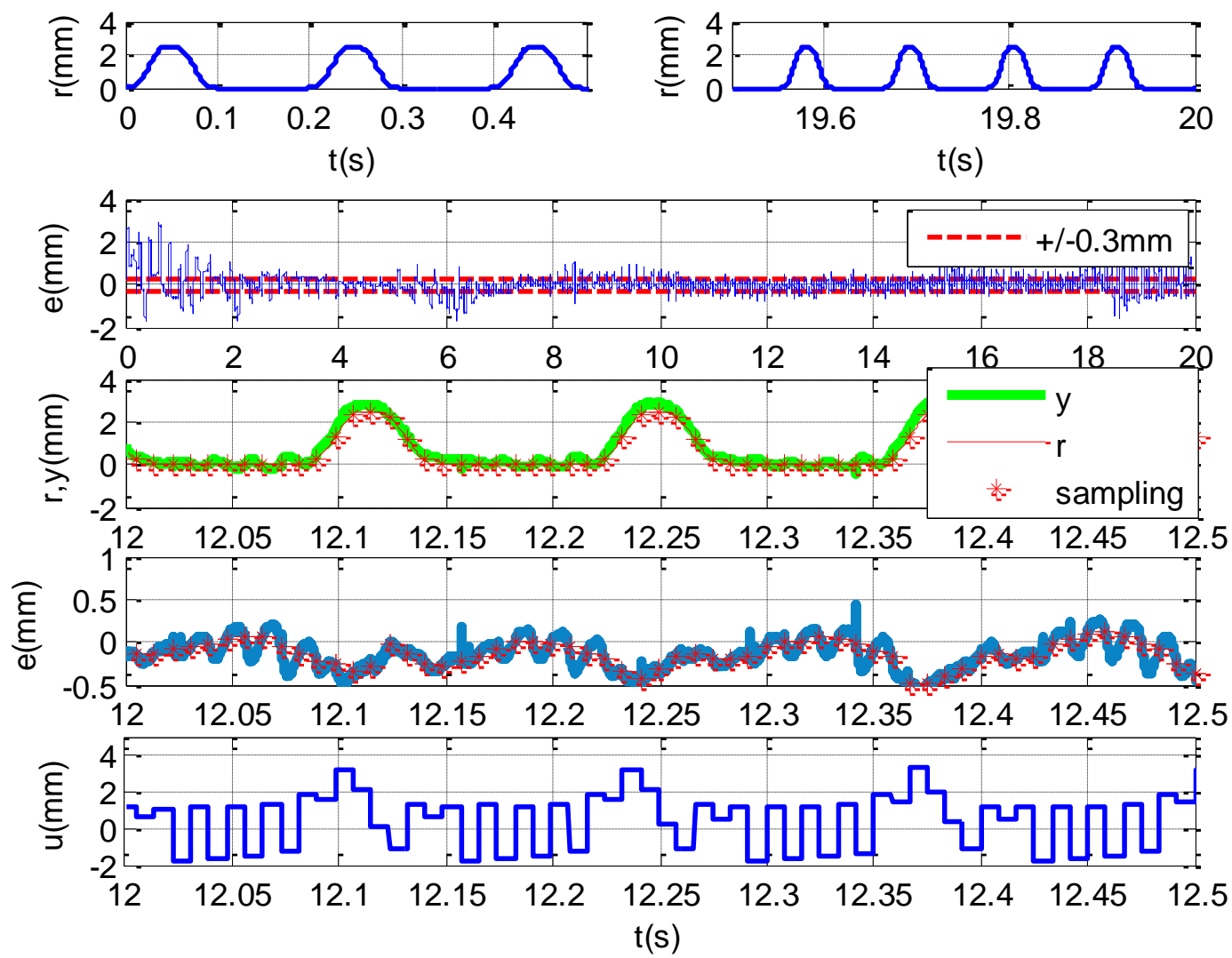

Figure 4.7. Experimental tracking results with 16-point fixed interval sampling $[5-9 \mathrm{~Hz} @$ $0.2 \mathrm{~Hz} / \mathrm{s}]$ with the convergence-improved stabilizer.

With the original stabilizer [15], the transient of 16-point fixed interval sampling is out of the implementation of the valve hardware and no experimental results are obtained. However, with the convergence-improved stabilizer, Figure 4.7 shows the tracking results. The error converges to $+/-0.3 \mathrm{~mm}$ within $3 \mathrm{~s}$ and the transient magnitude is $3 \mathrm{~mm}$. 


\subsubsection{Comparison}

The convergence time of the original stabilizer and the convergenceimproved stabilizer is compared in Table 4.1. It is clearly seen that for the specific number of sampling points, tracking error converges much faster with the convergence-improved stabilizer than with the original stabilizer. With the original stabilizer [15], convergence time increases dramatically while increasing the number of the sampling points. With the convergence-improved stabilizer, more sampling points have less effect on the convergence time.

Table 4.1: Comparison of the convergence achieved using the original stabilizer and convergence-improved stabilizer

\begin{tabular}{|c|c|c|c|c|}
\hline & \multicolumn{4}{|c|}{ Convergence time [s] } \\
\hline Number of sampling points & 10 & 12 & 14 & 16 \\
\hline The original stabilizer & 6 & 10.7 & 20.4 & -- \\
\hline $\begin{array}{c}\text { Convergence-improved } \\
\text { Stabilizer }\end{array}$ & 1.2 & 1.8 & 1.8 & 2.1 \\
\hline
\end{tabular}

\subsection{Experiment Validation for Variable Sampling in the Angle Domain}

Chapter 3 uses 12-point fixed and variable interval samplings as an example to show the improvement on the tracking performance by iterative sampling interval searching. In this section, more experimental tracking results with different numbers of sampling points $(4,6,8,10$ and 14 points) further validate its effectiveness. 


\subsubsection{4-point variable interval sampling case}

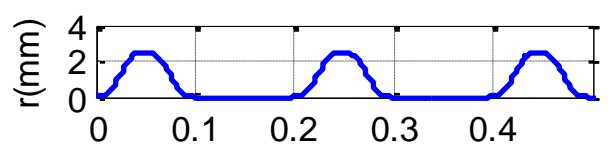

$\mathrm{t}(\mathrm{s})$

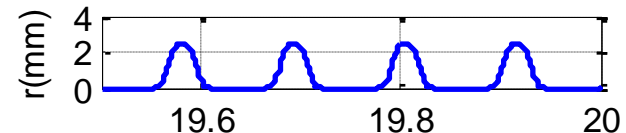

$\mathrm{t}(\mathrm{s})$
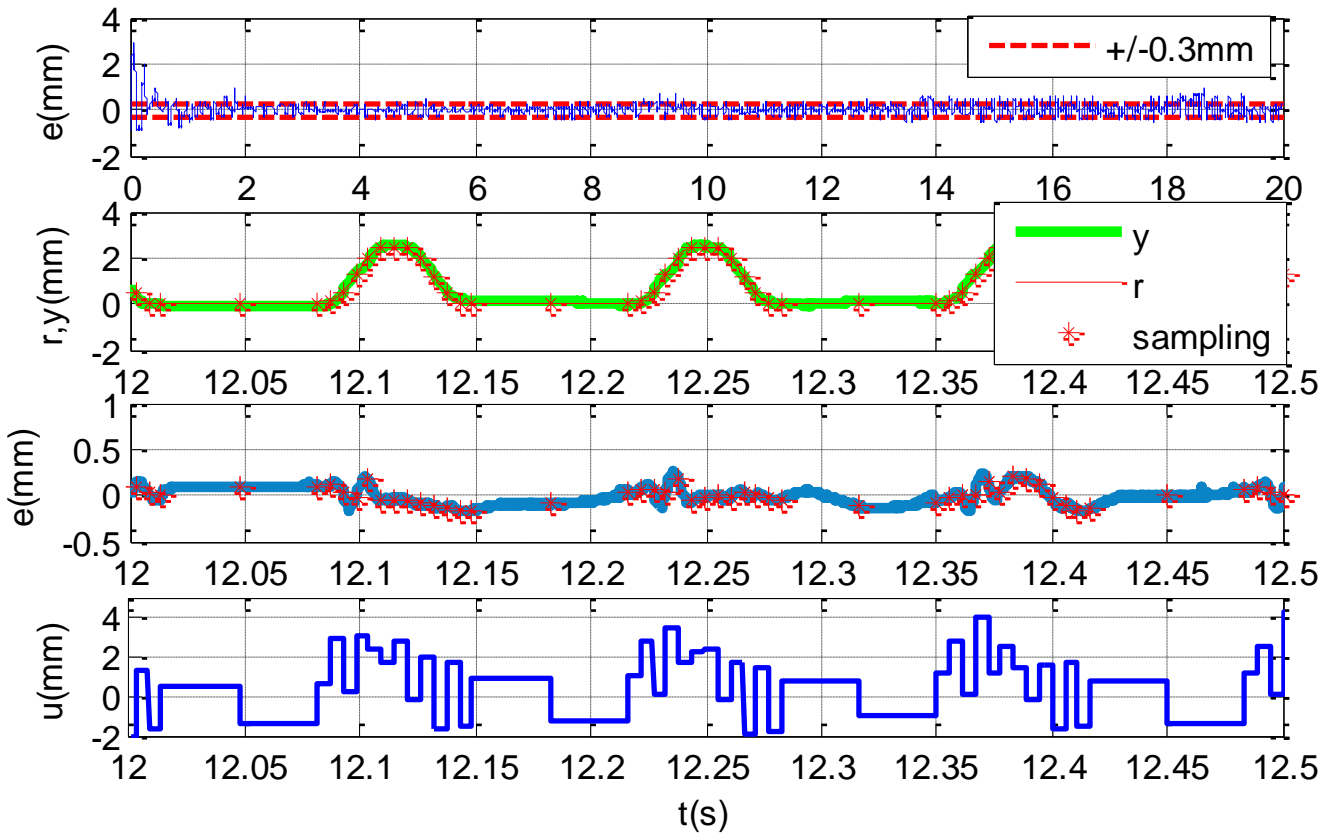

Figure 4.8. Experimental tracking results with 14-point variable interval sampling [5-

$9 \mathrm{~Hz} @ 0.2 \mathrm{~Hz} / \mathrm{s}]$ with the convergence-improved stabilizer.

Tracking performance with 14-point variable interval sampling by iterative sampling interval searching is shown in Figure 4.8. The desired variable interval sampling pattern $\theta_{\text {final }}$ is obtained as: $\left[0^{\circ}, 33.6694^{\circ}, 61.4249^{\circ}, 88.0086^{\circ}\right.$, $114.7995^{\circ}, 150.0000^{\circ}, 180.0000^{\circ}, 214.3019^{\circ}, 242.7658^{\circ}, 271.0510^{\circ}, 297.6666^{\circ}$, $330.0000^{\circ}, 360.0000^{\circ}, 540.0000^{\circ}$ ] by the variable sampling interval search in Chapter 3. Compared with the 14-point fixed interval sampling with sampling resolution $\Delta \theta=51.4286^{\circ}$ per revolution in both Figure 4.5 and Figure 4.6, the tracking error is within $+/-0.2 \mathrm{~mm}$, which is a little less than $+/-0.3 \mathrm{~mm}$ in both 
Figure 4.5 and Figure 4.6. The tracking performance is not dramatically improved for this high number of the sampling point case. The effectiveness of the variable sampling interval search in Chapter 3 is continuously tested on smaller number of the sampling point cases.

\subsubsection{0-point variable interval sampling case}

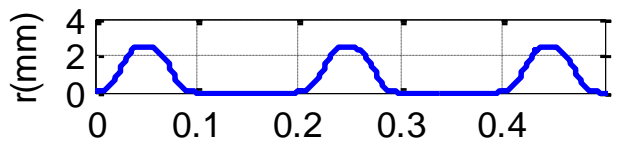

$\mathrm{t}(\mathrm{s})$

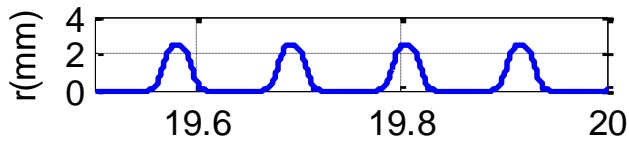

$\mathrm{t}(\mathrm{s})$
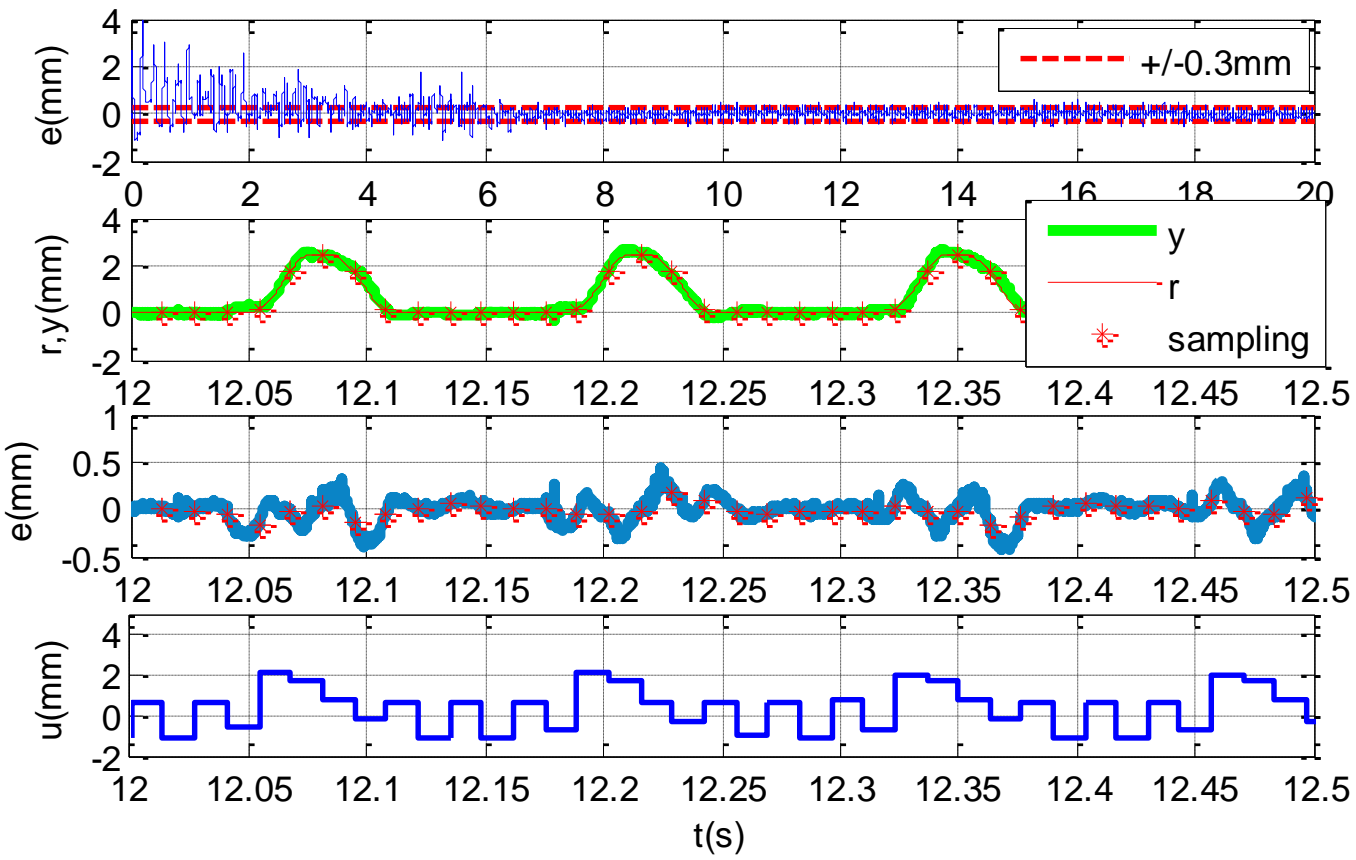

Figure 4.9. Experimental tracking results with 10-point fixed interval sampling $[5-9 \mathrm{~Hz} @$ $0.2 \mathrm{~Hz} / \mathrm{s}]$ with the original stabilizer.

Experimental tracking results with 10-point fixed and variable interval sampling are shown in Figure 4.9 and Figure 4.10 respectively. In Figure 4.9, the fixed sampling interval is $45^{\circ}$. The desired variable interval sampling pattern 
$\theta_{\text {final }}$ in Figure 4.10 is obtained as: $\left[0^{\circ}, 43.2300^{\circ}, 75.8501^{\circ}, 109.6141^{\circ}\right.$, $180.0000^{\circ}, 221.8935^{\circ}, 269.0651^{\circ}, 312.6740^{\circ}, 360.0000^{\circ}, 540.0000^{\circ}$. It is clearly seen that the tracking error of the steady state during $7-9 \mathrm{~Hz}(10-20 \mathrm{~s})$ in Figure 4.10 is within $+/-0.2 \mathrm{~mm}$, which is much less than the error range $+/-0.5$ of the steady error during $7-9 \mathrm{~Hz}$ in Figure 4.9. The RMS error of the tracking error in Figure 4.10 during $7-9 \mathrm{~Hz}$ is $0.1 \mathrm{~mm}$, which is also much less than $0.23 \mathrm{~mm}$ (the RMS error with the 10-point fixed sampling). Moreover, the convergence time in Figure 4.10 with the convergence-improved stabilizer is much less than the time in Figure 4.9 with the original stabilizer.

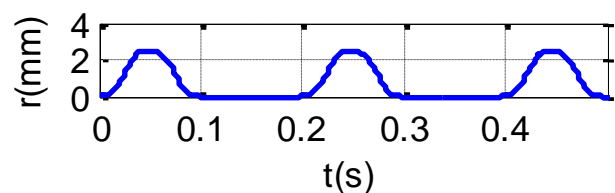

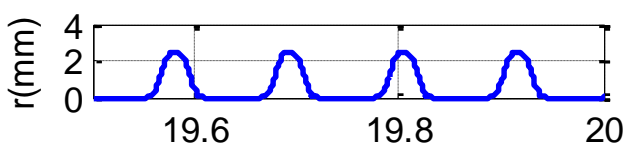

$\mathrm{t}(\mathrm{s})$
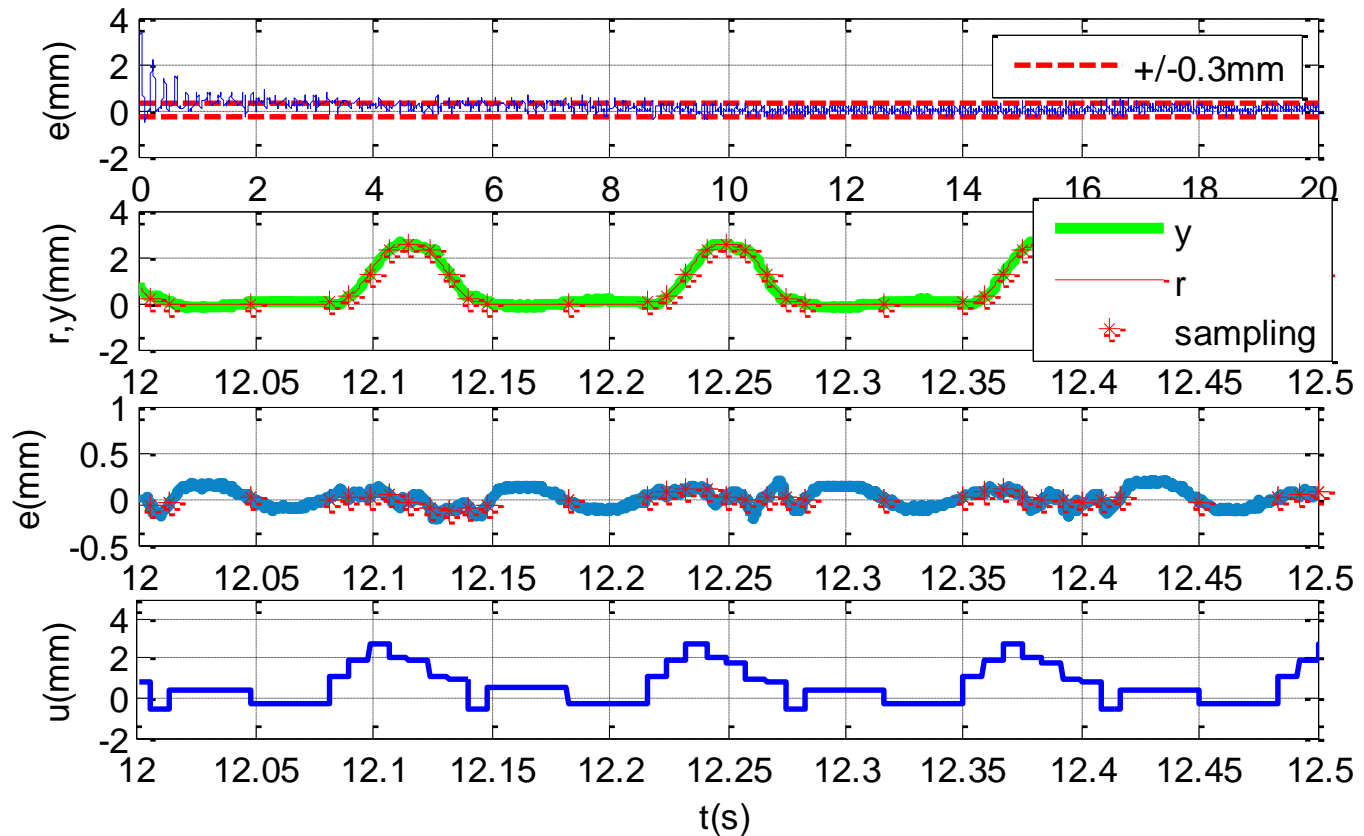

Figure 4.10. Experimental tracking results with 10-point variable interval sampling [5$9 \mathrm{~Hz} @ 0.2 \mathrm{~Hz} / \mathrm{s}]$ with the convergence-improved stabilizer. 


\subsubsection{8-point variable interval sampling case}
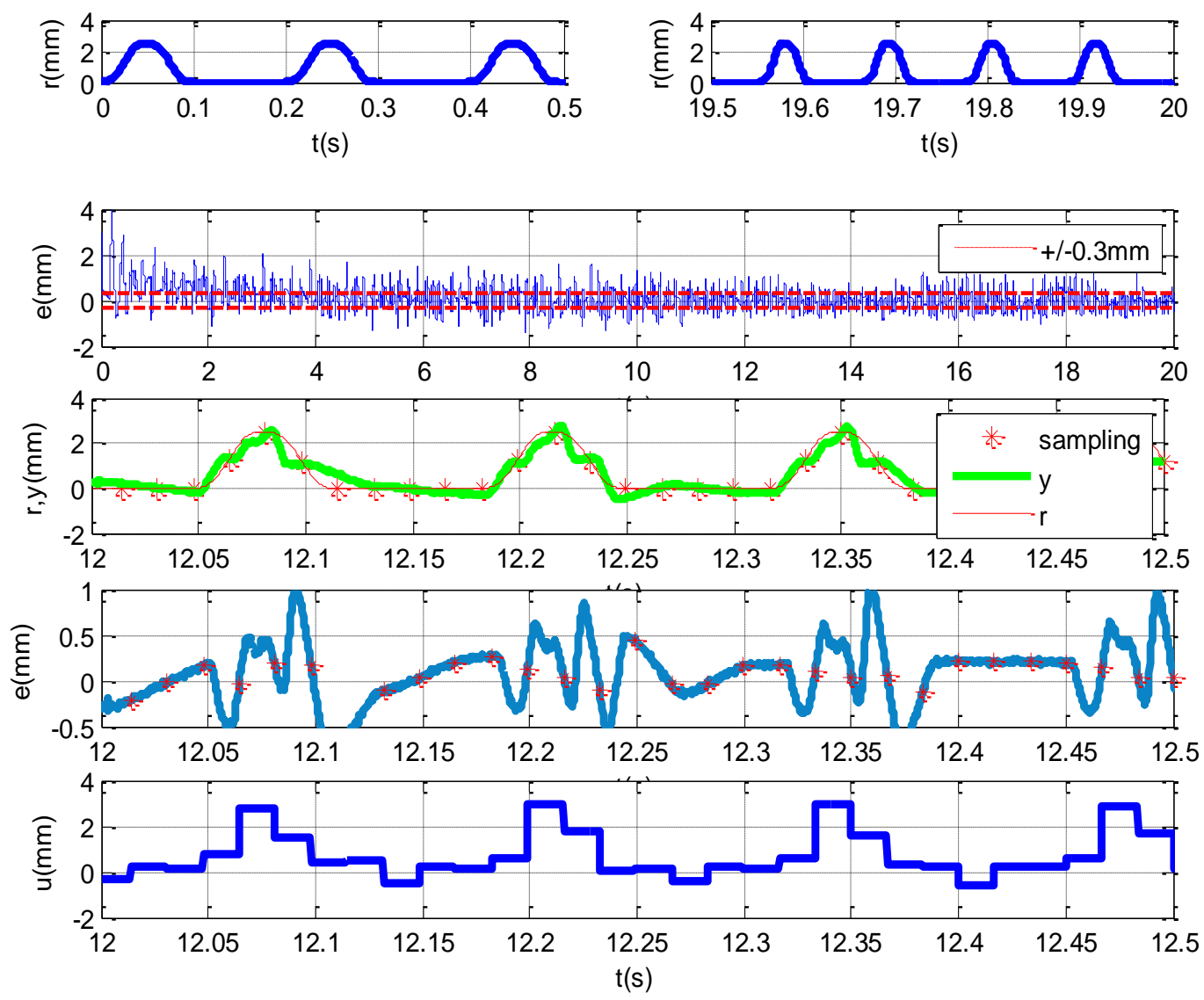

Figure 4.11. Experimental tracking results with 8-point fixed interval sampling $[5-9 \mathrm{~Hz} @$ $0.2 \mathrm{~Hz} / \mathrm{s}]$ with the original stabilizer.

With the 8-point fixed interval sampling in Figure 4.11, the tracking error is within the range from -0.5 to $1 \mathrm{~mm}$, which is much larger than the tracking error with 10-point fixed interval sampling. With the benefit of the iterative sampling interval searching in Chapter 3, the tracking performance is improved to limit the error within $+/-0.2 \mathrm{~mm}$ in Figure 4.12, which is similar to the error range with the 10 -point variable sampling case. Although the convergence-improved stabilizer is 
applied to obtain Figure 4.12, there is no obvious improvement on the convergence time. In Figure 4.11, the tracking error also goes into the steady state within $2 \mathrm{~s}$, although the steady error in Figure 4.11 has much larger error range. Thus, the effectiveness of the convergence-improved stabilizer can be clearly seen for higher order of the internal model based system, which is corresponding to the larger number of the sampling points per revolution.
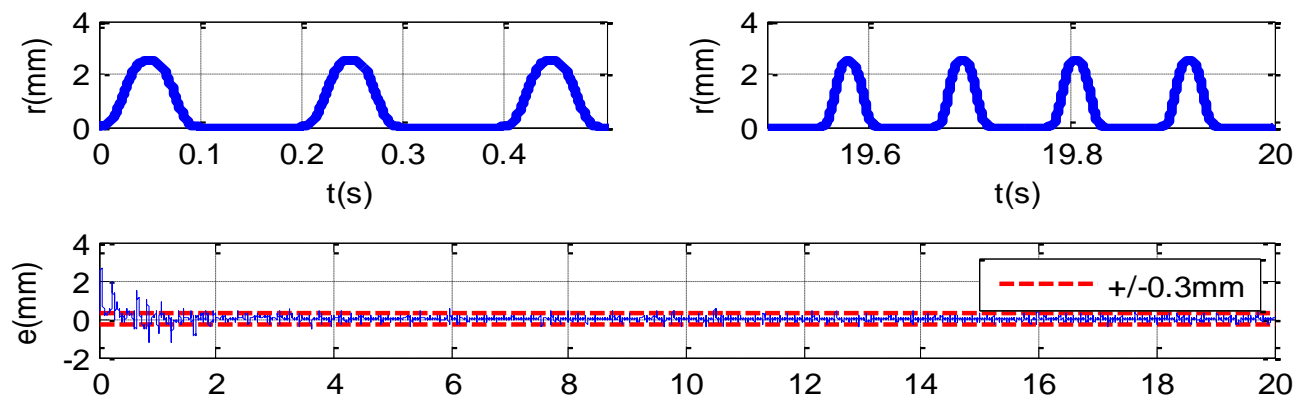

$\mathrm{t}(\mathrm{s})$
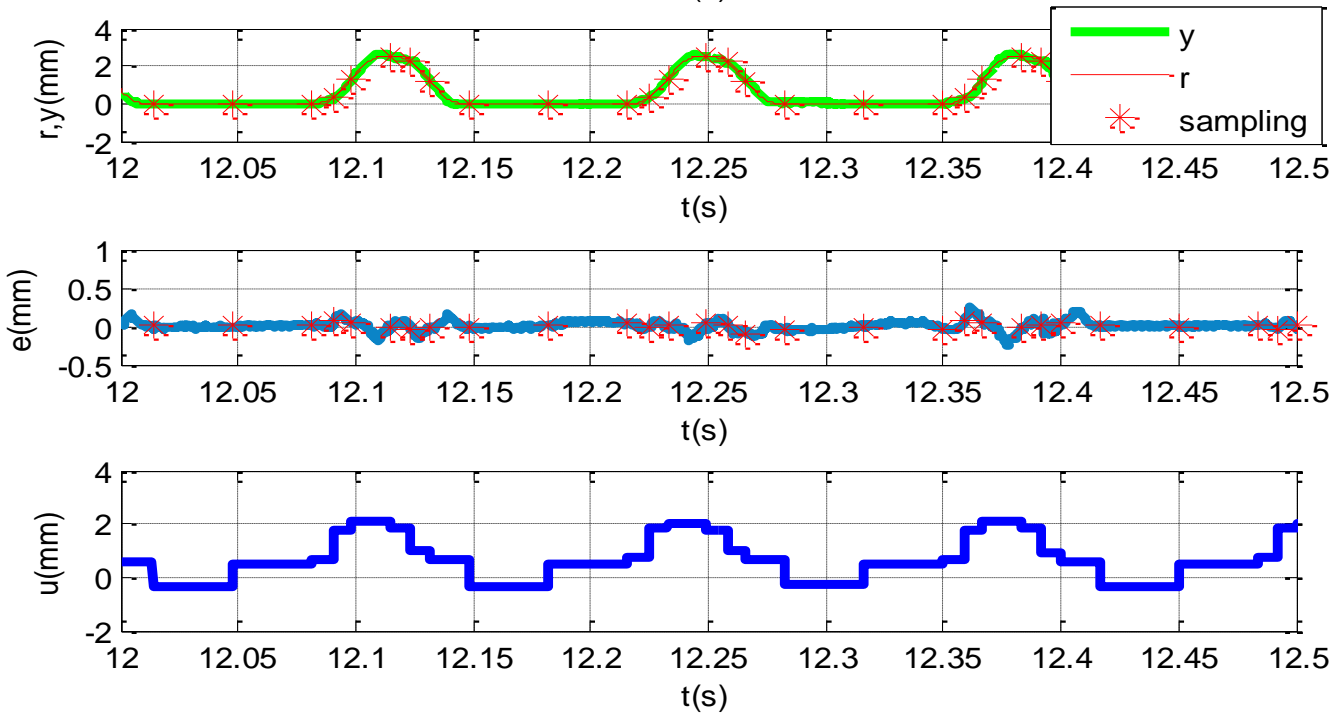

Figure 4.12. Experimental tracking results with 8-point variable interval sampling $[5-9 \mathrm{~Hz}$ @ $0.2 \mathrm{~Hz} / \mathrm{s}]$ with the convergence-improved stabilizer. 


\subsubsection{6-point and 4-point variable interval sampling cases}

When the number of the sampling points is less than 8 , the improvement of the tracking performance by variable sampling interval search can be clearly observed by the comparison of tracking results with the fixed and variable interval sampling cases.

For the 4-point and 6-point fixed interval sampling cases, only 3 sampling points are located to the desired lift portion of the reference profile, which are not enough to provide a fine sampling resolution. The tracking error with 6-point fixed interval sampling in Figure 4.13 is within the range from $-0.7 \mathrm{~mm}$ to $2 \mathrm{~mm}$, which is almost double of the error range with 8-point fixed interval sampling in Figure 4.11. In Figure 4.15, the tracking error with 4-point fixed interval sampling is within the range from $-3 \mathrm{~mm}$ to $3 \mathrm{~mm}$, which is much worse than the tracking performance of any larger number of sampling points.

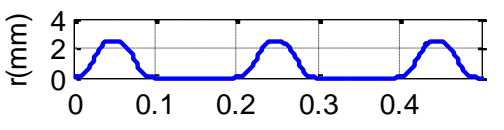

$\mathrm{t}(\mathrm{s})$
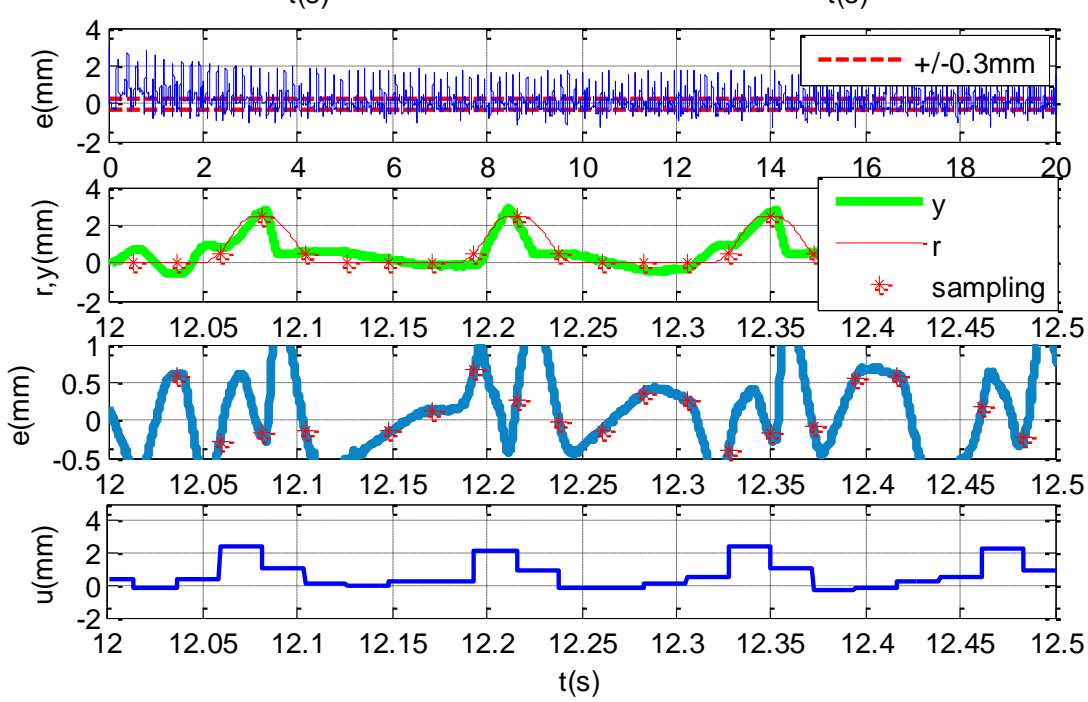

Figure 4.13. Experimental tracking results with 6-point fixed interval sampling $[5-9 \mathrm{~Hz} @$ $0.2 \mathrm{~Hz} / \mathrm{s}]$ with the original stabilizer. 


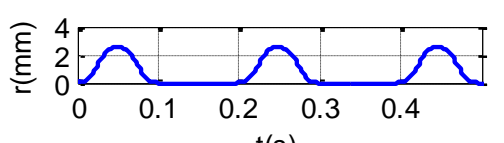

$\mathrm{t}(\mathrm{s})$

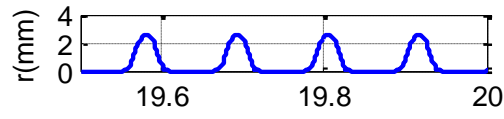

$\mathrm{t}(\mathrm{s})$
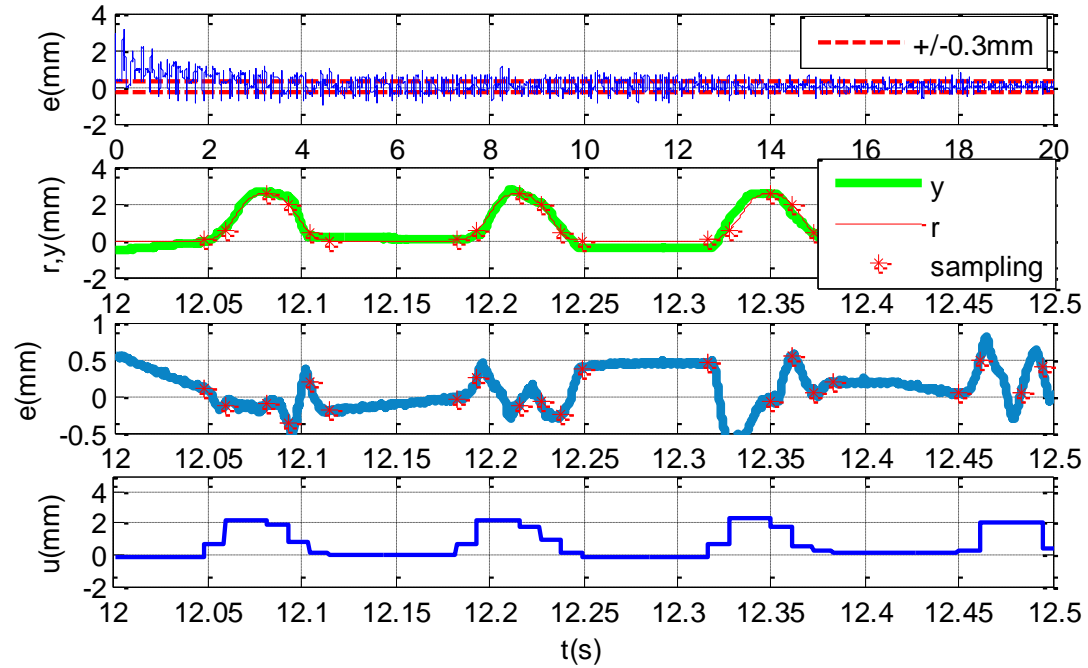

Figure 4.14. Experimental tracking results with 6-point variable interval sampling $[5-9 \mathrm{~Hz}$ $@ 0.2 \mathrm{~Hz} / \mathrm{s}]$ with the originl stabilizer.

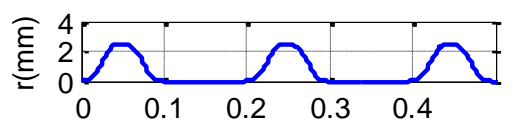

$\mathrm{t}(\mathrm{s})$

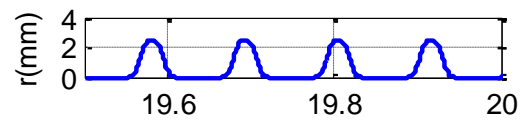

$\mathrm{t}(\mathrm{s})$
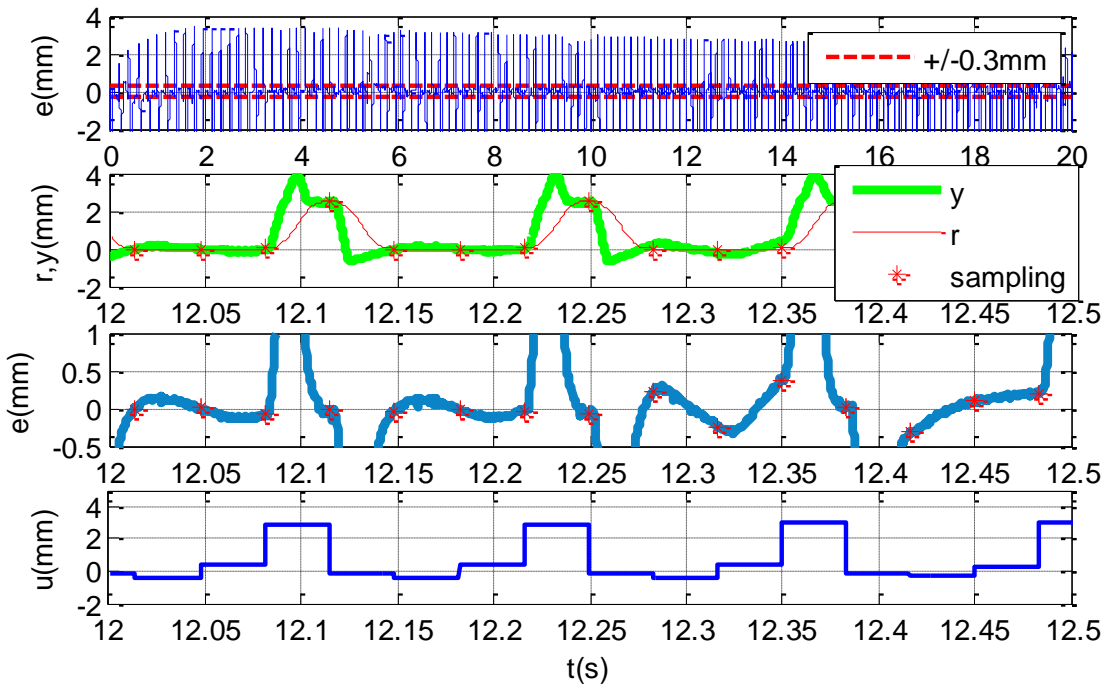

Figure 4.15. Experimental tracking results with 4-point fixed interval sampling $[5-9 \mathrm{~Hz} @$ $0.2 \mathrm{~Hz} / \mathrm{s}]$ with the originl stabilizer. 


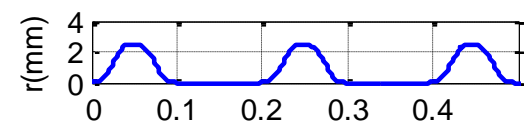

$\mathrm{t}(\mathrm{s})$

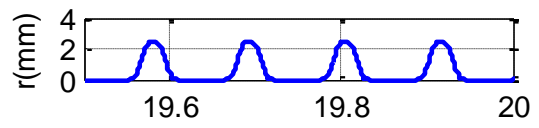

$t(s)$
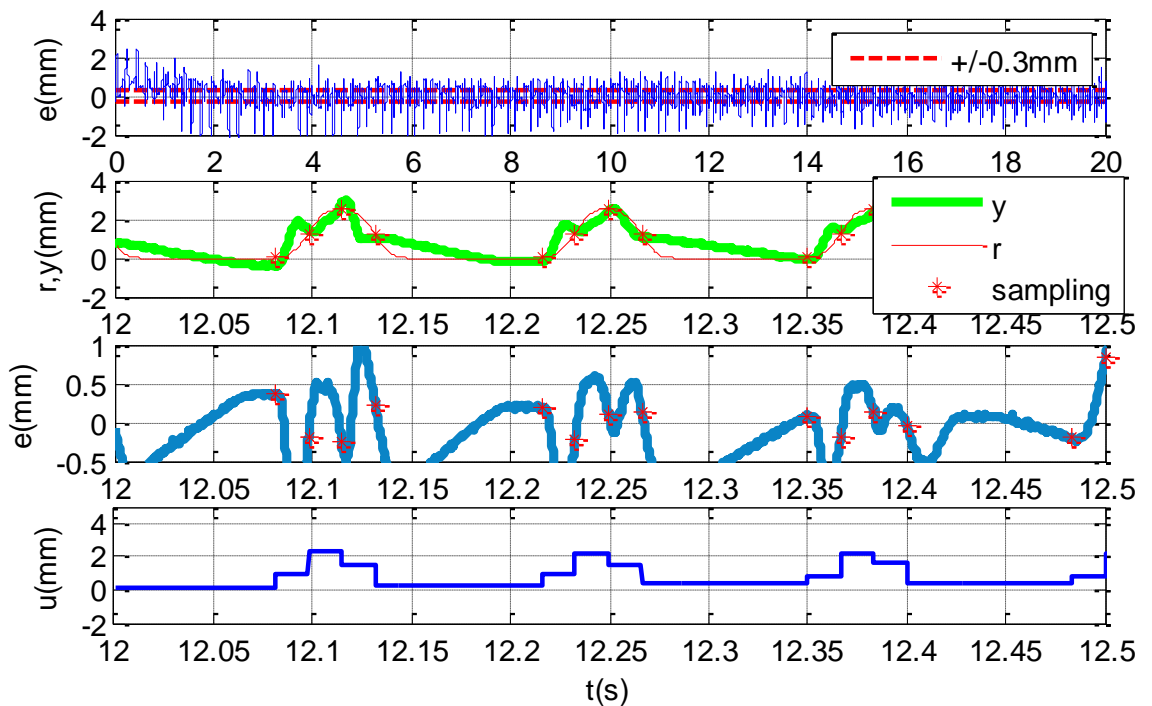

Figure 4.16. Experimental tracking results with 4-point variable interval sampling $[5-9 \mathrm{~Hz}$ @ $0.2 \mathrm{~Hz} / \mathrm{s}$ ] with the originl stabilizer.

Applied the variable sampling interval search in Chapter 3, the tracking error with 6-point variable interval sampling can be improved to the range of $+/-0.3 \mathrm{~mm}$ finally (shown in Figure 4.14, second plot after 14s). The tracking error with 4point variable interval sampling in Figure 4.16 can be reduced to $+/-1 \mathrm{~mm}$, which is much better than $+/-3 \mathrm{~mm}$ in Figure 4.15 . The original stabilizer is used in 6point and 4-point variable interval sampling cases.

\subsection{Comparison Analysis}

The comparison of tracking performance using different numbers of sampling points per revolution is shown in Figure 4.17. Both the RMS and peak values of the steady state of the tracking error are significantly improved using the 
proposed variable interval sampling search in Chapter 3 . It can be clearly seen that the corresponding tracking errors decrease with the increasing number of sampling points. However, for the variable interval sampling case, the error decrease is no longer significant when the number of sampling points is beyond 8. As the number of sampling per revolution determines the internal model order, using 8-point sampling per revolution should be sufficient for this particular tracking problem.
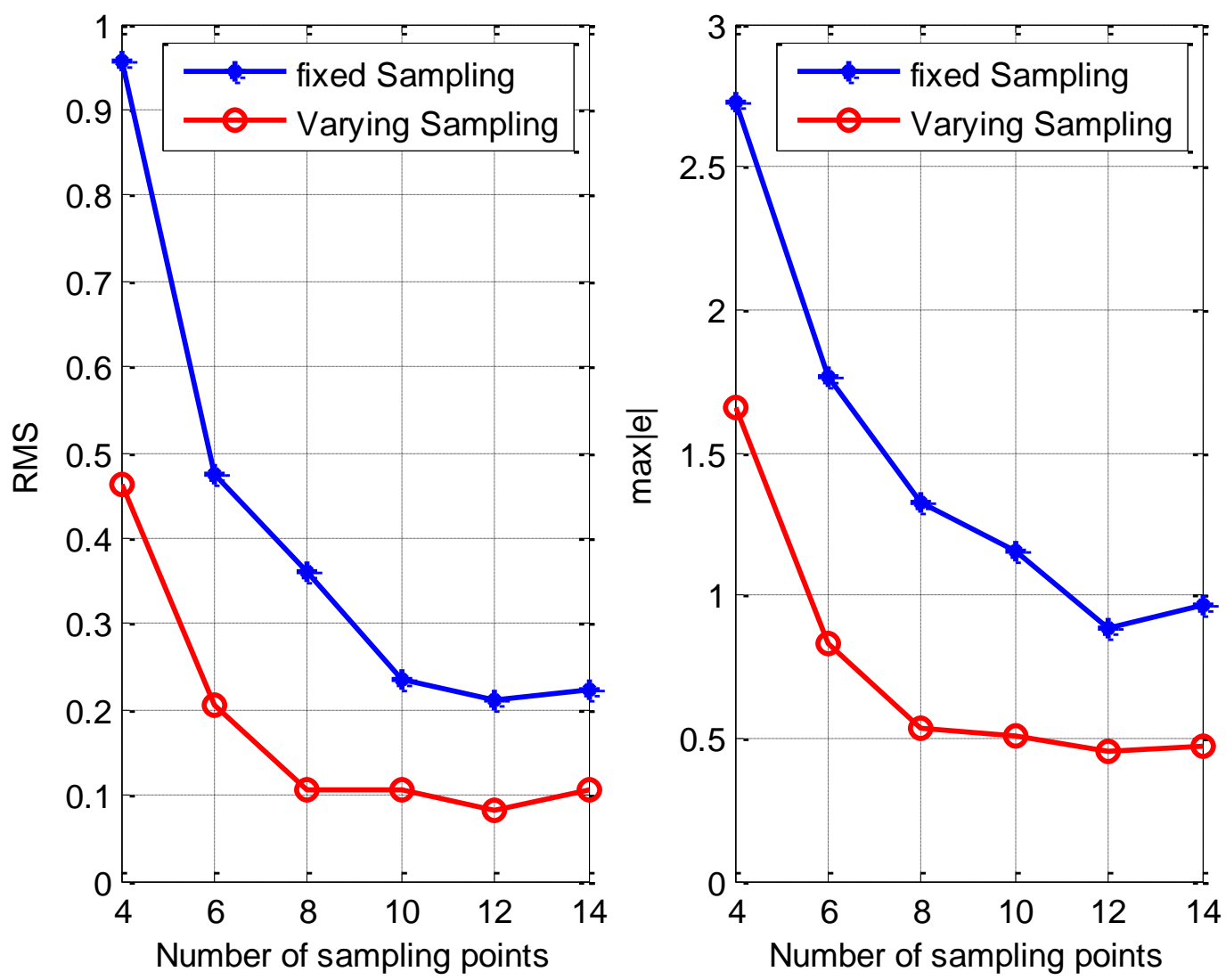

Figure 4.17. Comparison of the tracking performance achieved using different internal model unit during the steady states. 

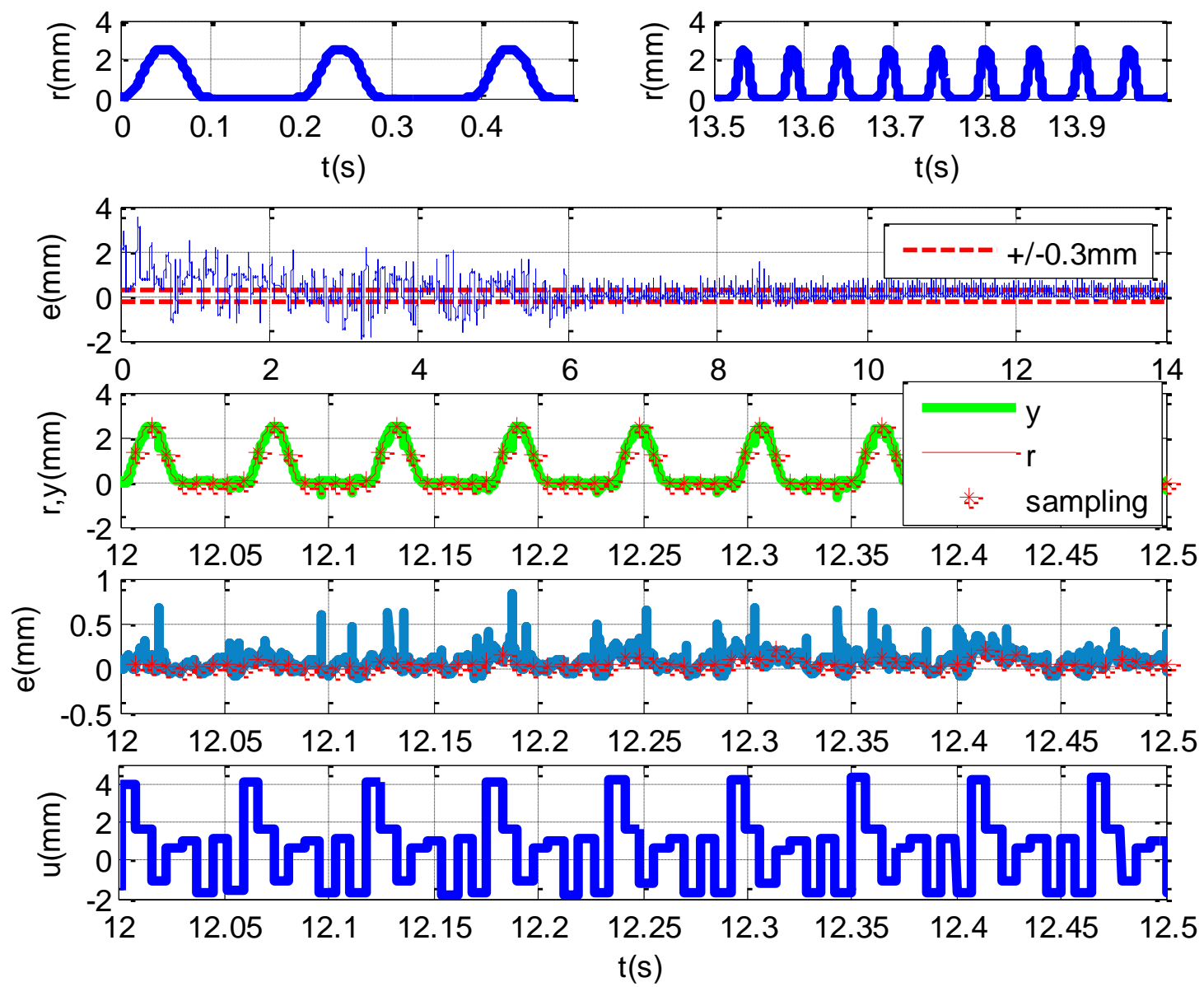

Figure 4.18. Experimental tracking results with 8-point fixed interval sampling $[5-20 \mathrm{~Hz}$ @ $1 \mathrm{~Hz} / \mathrm{s}]$ with the original stabilizer.

The experimental comparison results in Figure 4.17 show that 8 -point variable interval sampling design can provide a good balance between the complexity of the internal model unit and the precise tracking performance for this application. The experimental result with 8-point fixed sampling interval is shown in Figure 4.11, and the one with 8-point variable interval samplings is shown in Figure 4.12. The frequency variation rate for both cases are $0.2 \mathrm{~Hz} / \mathrm{sec}$. Again, the tracking improvement using variable interval sampling is very evident. 
In addition, to validate the proposed method under different frequency variation rates, the experimental results with $1 \mathrm{~Hz} / \mathrm{sec}$ variation rate is also shown in Figure 4.18 and Figure 4.19 for fixed and variable interval sampling respectively. The improved tracking result in Figure 4.19 once again demonstrates the effectiveness of the proposed interval searching method.

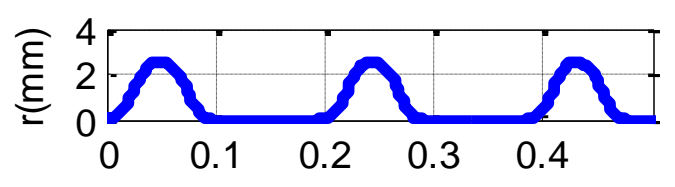

$\mathrm{t}(\mathrm{s})$

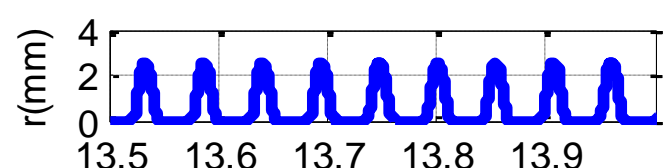

$\mathrm{t}(\mathrm{s})$
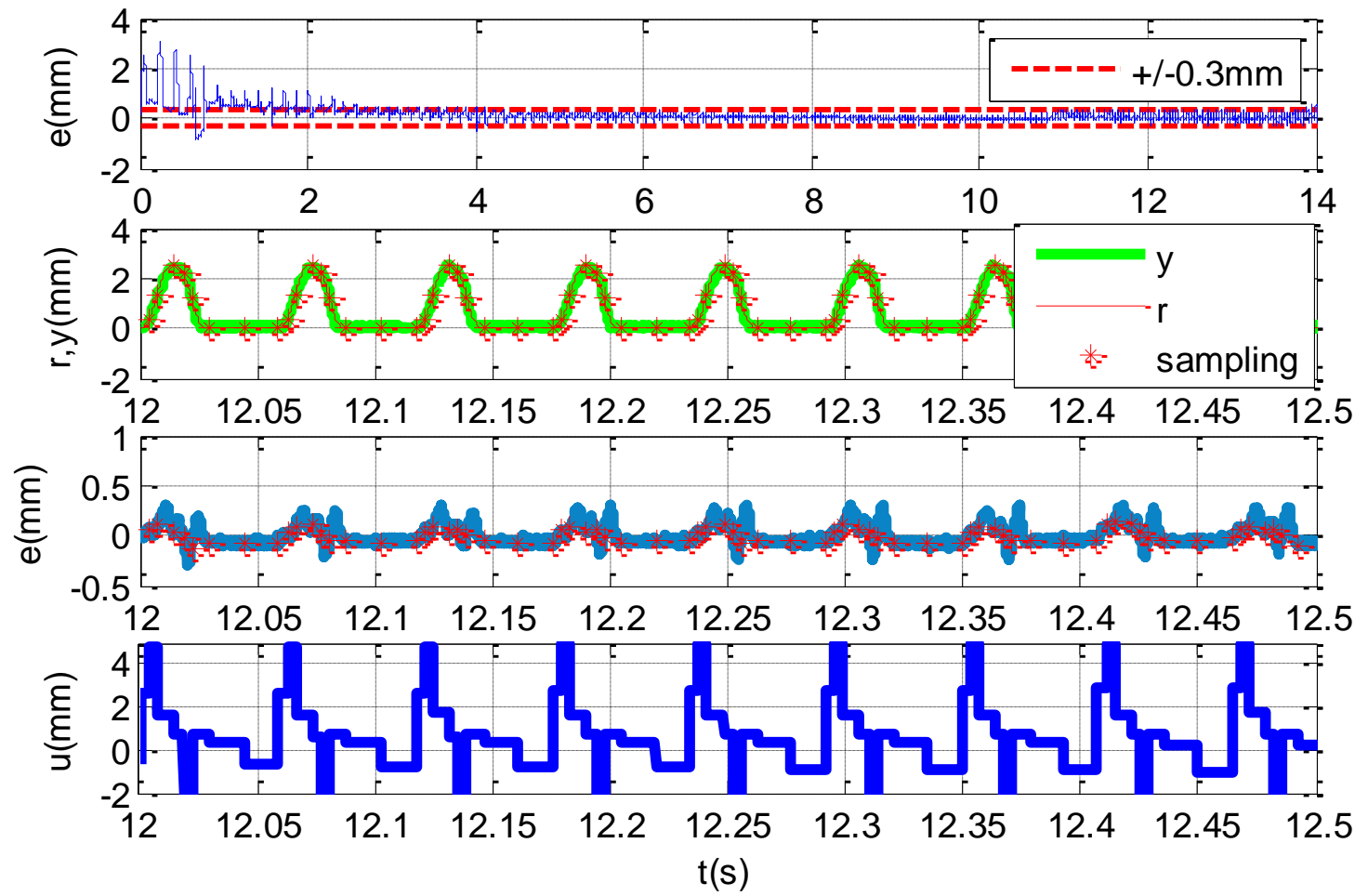

Figure 4.19. Experimental tracking results with 8-point variable interval sampling [5$20 \mathrm{~Hz} @ 1 \mathrm{~Hz} / \mathrm{s}]$ with the convergence-improved stabilizer. 


\subsection{Conclusion}

This chapter investigates the experimental results from a time-varying internal model based camless engine valve actuation system and demonstrates the effectiveness of the proposed methods. A quantitative analysis helps to highlight the strength of the variable interval sampling on less computational complexity and better tracking performance. 


\section{Chapter 5}

\section{Conclusion}

This work investigates the reference tracking/disturbance rejection based on the internal model principle in the rotational angle domain. Motivated by the fact that the signal of interest is aperiodic in time domain but periodic with respect to the rotational angle, the plant dynamics is converted into the angle domain and becomes angle varying. Two particular issues are addressed to enable practical implementation. First, to improve the control convergence rate, additional LMI constraints are added to the existing stabilizer synthesis approach, so that the tracking error can quickly converge to steady state. Second, to limit the number of sampling per revolution while maintaining tracking accuracy, a varying interval sampling method is proposed, where the location of sampling points can be optimized based on an iterative searching method. The control approach is then validated using a camless engine valve actuation system. 


\section{References}

[1] J.W. Kim, et. al. Chemo-sensitivity and reliability of flagellar rotary motor in a MEMs microfluidic actuation system. Sensor and Actuators B (Chemical): Vol. 114 (1): pp. 229-238, 2006.

[2] M. Nakano, L.-H. She, Y. Mastuo, and T. Hino. Elimination of position-dependent disturbances in constant speed rotation control systems. Control Engineering Practice, 4(9):1241-1248, 1996.

[3] T.-C. Tsao and K. C. Pong. Control of radial run out in multi-tooth face milling. Transactions of the North American Manufacturing Research Institute of SME, pages 183-190, 1991.

[4] Jin-Hua She and M. Nakano, 1996. "Elimination of position-dependent disturbances in constant-speed-rotational control systems - a nonlinear compensation approach". Proceeding of the 35th IEEE Conference on Decision and Control, Vol 3, pp. 27012706.

[5] Sun, Z., Cleary, D., 2003. "Dynamics and control of an electro-hydraulic fully flexible valve actuation system". Proceeding of the American Control Conference, Denver, CO, pp. $3119-3124$.

[6] Y.-K. Chin and F. E. Coats. Engine dynamics: Time-based versus crank-angle based. In SAE Technical Paper Series 860412, 1986.

[7] P. G. Scotson and W. P. Heath. Crank-angle based control of high speed diesel engines. In SAE Technical Paper Series 1996-06-19, 1996.

[8] S. Yurkovich and M. Simpson. Comparative analysis for idle speed control: A crankangle domain viewpoint. Proceedings of the 1997 American Control Conference, vol. 1, pp 278-283, Albuquerque, NM, USA, 1997.

[9] Junping Wang and T.-C. Tsao, 2004. "Repetitive control of Linear Time Varying System with Application to Electronic Cam Motion Control". Proceeding of the 2004 American Control Conference, vol.4, pp. 3794 - 3799. 
[10] Junqing Wang and T. C. Tsao, 2005. "A Two Parameter Robust Linear Parameter Varying Repetitive Control Approach to Variable Speed Electronic CAM Follower Problem". ASME 2005 International Mechanical Engineering Congress and Exposition, Paper No. IMECE2005-82904, pp. 109-114.

[11] Zongxuan Sun, 2004. "Tracking or rejecting rotational-angle dependent signals using time varying repetitive control". Proceeding of the 2004 American Control Conference, vol.1, pp. 144- 149.

[12] C.-L. Chen and G.T.-C. Chiu, "Spatially Periodic Disturbance Rejection with Spatially Sampled Robust Repetitive Control," ASME Journal of Dynamic Systems, Measurement and Control, Vol. 130, Iss. 2, pp. 021002-1-021002-11, March 2008

[13] Zhang, Z., and Sun, Z., 2010. "A novel internal model-based tracking control for a class of linear time-varying systems". Journal of Dynamic Systems, Measurement, and Control, Vol. 132, 011004.

[14] Gillella, P., Song, X., and Sun, Z., 2014. "Time-varying internal model based control of a camless engine valve actuation system". IEEE Trans. Control System Technology, Vol. 22, Iss. 4, pp. 1498-1510

[15] Song, X., Gillella, P., and Sun, Z., 2012. "Robust stabilization of discrete linear time varying internal model based system". Proceedings of the 2012 ASME Dynamic Systems and Control Conference, Paper No. DSCC2012-MOVIC2012-8745, pp. 153-161

[16] Song, X., Gillella, P. and Sun, Z.,2013. "A New Stabilizer for LTV Internal Model Based System And Its Application To Camless Engine Valve Actuation", Proceedings of the 2013 American Control Conference, Washington, DC, pp.5284-5289

[17] Turner, J. W. G., Bassett, M. D., Pearson, R. J., Pitcher, G., and Douglas, K. J., 2004. "New operating strategies afforded by fully variable valve trains". SAE Technical Paper, 2004-01-1386.

[18] Kitabatake, R., Minato, A., Inukai, N., and Shimazaki , N., 2011. "Simultaneous improvement of fuel consumption and exhaust emissions on a multi-cylinder camless engine". SAE Technical Paper Series, pp 2011-01-0937.

[19] Wen-An Zhang, Li Yu, 2009. "Stability analysis for discrete-time switched timedelay system". Automatica, Volume 45, Issue 10, pp. 2265-2271. 